\title{
MESON EXCHANGE POTENTIALS IN AN NN QUARK MODEL WITH QUARK-ANTIQUARK EXCITATIONS
}

\author{
Y. FUJIWARA and K.T. HECHT \\ Physics Department, University of Michigan, Ann Arbor, MI 48109, USA ${ }^{\dagger}$
}

Received 25 June 1985

(Revised 7 October 1985)

\begin{abstract}
In a recent quark-model study of the NN interaction, we explicitly incorporated the qq excitations inherent in the quark-gluon interaction lagrangian into the model space, and examined the contributions to the NN potential of particular $(3 q)(q \bar{q})$ components of the single-nucleon wave function, with specific baryon and meson quantum numbers, through the Wigner transforms of the resultant coupling terms of the quark exchange kernels. The present investigation focuses on the long-range parts of the potentials. It is shown that these arise almost entirely through two of the 25 possible types of exchange terms, and that these two correspond to the simple exchange of a $q \bar{q}$ pair between the two nucleons and contribute only through coupling terms for which the (3q) components have the quantum numbers of a nucleon and the ( $q \bar{q})$ component the color-singlet character of a real pseudoscalar or vector meson. The potentials arising from these simple colorsinglet $q \bar{q}$ exchanges have been evaluated in explicit analytic form. In their dependence on nucleon $\left(\sigma_{1} \cdot \sigma_{2}\right)$ and $\left(\tau_{1} \cdot \tau_{2}\right)$ factors and the relative importance and signs of spin-spin, spin-independent central, $L S$, and tensor terms they have all the characteristics of conventional OBEP's. They are in remarkably good agreement with conventional OBEP's in the $r \geqslant 1.2 \mathrm{fm}$ range and have the same qualitative radial features over an even wider range, the one exception being the steep short-range repulsive rise of the spin-independent central term of the $\omega$-exchange potential. The corresponding $q \bar{q}$ exchange potential instead changes sign to become attractive at $r<0.8 \mathrm{fm}$.
\end{abstract}

\section{Introduction}

In the past few years QCD-inspired quark models have been widely used in attempts to gain a more fundamental understanding of the NN interaction. In the simplest quark models the nucleon is assumed to be a pure three-quark (3q) system. The earlier studies of the $N N$ interaction based on such simple (3q)-(3q) models have attempted only the elucidation of the characteristics of the extreme short-range repulsive part of this interaction. In a recent study ${ }^{1}$ ) we explicitly incorporated the quark-antiquark excitations inherent in the quark-gluon interaction lagrangian into a quark model to study the effects of such excitations on the NN interaction. In this study the (3q) components of the single-nucleon wave function were augmented by $(3 q)(q \bar{q})$ components generated by the off-shell terms of the Fermi-Breit onegluon exchange interaction. All 24 possible spin, isospin, color combinations of the $(3 q)(q \bar{q})$ excitations with the resultant quantum numbers of a single nucleon and energetically lowest p-wave relative motion functions were included. Quark exchange

† Supported by the US National Science Foundation. 
kernels for the two-nucleon system described by these improved single-nucleon internal wave functions were calculated in the framework of the resonating group method. These exchange kernels were then transformed into momentum-dependent potentials and converted into phase-shift-equivalent effective $\mathrm{NN}$ potentials through a local-momentum approximation using the Wigner transforms of the kernels. The contributions to the exchange kernels from the $(3 q)(q \bar{q})$ components of the nucleon wave functions lead to the following effects in the S-wave NN potentials: the repulsive core heights of the simple (3q)-(3q) model are greatly reduced but retain their strong energy dependence; the potentials gain an attractive part in the $0.8-2 \mathrm{fm}$ range.

Since the $(q \bar{q})$ admixtures make significant contributions to the properties of the nucleon itself, these were examined in detail in ref. ${ }^{1}$ ). It was shown, in particular, that the improved single-nucleon wave functions are consistent with the low-energy electromagnetic properties of the nucleon and are consistent with the strengths of the nucleon-meson coupling constants for $\eta, \rho$ and $\omega$ mesons. Moreover, they give the observed tensor/vector coupling ratios for the vector mesons, and the vector dominance of the electromagnetic form factors is reproduced naturally. However, since a simple ( $\mathrm{q} \overline{\mathrm{q}})$ cluster with the quantum numbers of a pion cannot be expected to give a realistic picture of the pion, agreement with the observed strength of the pion-nuclcon coupling constant was not expected. (The predicted pion-nucleon coupling constant $g_{\mathrm{NN} \pi}^{2}$ is too weak by a factor of $\sim 3$.) Also, since a simple $(\mathrm{q} \overline{\mathrm{q}})$ cluster with the quantum numbers of a pion cannot be expected to have the mass of a real pion, we do not expect to reproduce the extreme long-range Yukawa tail of the OPEP part of the NN interaction.

Despitc the possible shortcomings of the extreme long-range part of the interaction, it was the aim of the study of ref. ${ }^{1}$ ) to explicitly incorporate the mesonic degrees of freedom into the quark models through such $(\mathrm{q} \overline{\mathrm{q}})$ excitations. By thus staying completely within the framework of a quark model it was hoped to bridge the gap between the simple quark model and more sophisticated models in which thc quark degrees of freedom are coupled to neson fields ${ }^{2}$ ) where, however, these meson fields are considered as separate entities outside the basic framework of QCD. Nevertheless, it was difficult to recognize the characteristics of the conventional meson exchange potentials in the NN potentials of ref. ${ }^{1}$ ). In principle, the Wigner transforms of the quark exchange kernels are ideal to study the contribution to the NN potential of a particular $(3 q)(q \bar{q})$ term with specific baryon and meson quantum numbers. Since the largest amplitudes of the $(3 q)(q \tilde{q})$ components in the improved single-nucleon wave function are of order $c \leqslant 0.25$, only terms of first order in these c's need to be retained in the calculation of the full exchange kernels for the two-nucleon system. It is thus possible to turn on one $(3 q)(q \bar{q})$ term at a time and examine the contribution corresponding to a $q \bar{q}-$ pair excitation with the color-singlet and spin, isospin characteristics of a particular real meson. Such curves are exhibited in ref. ${ }^{1}$ ); but since they gain their most prominent contributions in 
the $r \leqslant 1 \mathrm{fm}$ range, the discussion of ref. ${ }^{1}$ ) focused attention on the short-range parts of these potentials where the effects of antisymmetrization may also wash out the distinctions between different $(\mathrm{q} \bar{q})$ mesons. In general, these potentials gain contributions from 25 distinct types of exchange terms corresponding to 6 different types of quark exchanges each with as many as 5 distinct possible placements of the quark potential lines. The prominent extreme short-range parts of these potentials have their origin in the more complicated exchange terms of the "three-cluster" variety, i.e. terms involving quark exchanges between the quark of the qq pair as well as quarks from the $(3 q)$ clusters of both nucleons. They thus correspond to processes more complicated than the simple exchange of a q $\bar{q}$ pair between the two nucleons. To make the connection between these potentials and the conventional one-boson exchange potentials it is necessary to isolate those exchange terms which actually correspond to the exchange of a $q \bar{q}$ pair between the two nucleons. It is the purpose of this investigation to show that the long-range parts $(r \geqslant 1.2 \mathrm{fm})$, of the effective NN potential of ref. ${ }^{1}$ ) are dominated by two of the 25 types of quark exchange terms, that these exchange terms correspond to the exchange of a $q \bar{q}$ pair, and that the simple color singlet qq̄-pair exchange terms of this type lead to NN potentials which are in very good qualitative agreement with the usual one-meson exchange potentials. The simple $q \bar{q}$-pair exchange terms involve only quark exchanges of the "two-cluster" variety, i.e. the exchange of the quark of the qq pair in one nucleon with a quark from a single (3q) cluster in the other nucleon. Such exchange terms have been named ${ }^{3}$ ) "semi-direct". The momentum dependence of these simple color-singlet $q \bar{q}$ exchange terms is very mild compared with the strong momentum dependence of the full Wigner transforms including all 25 types of exchange terms. The smaller degree of nonlocality of the simple color-singlet $q \bar{q}$ exchange terms makes it even more attractive to identify these with the meson exchange potentials. Finally, the simplicity of the color-singlet $q \bar{q}$ exchange terms has made it possible to evaluate the exchange kernels for these terms, as well as their Wigner transforms, in explicit analytic form. It is also possible to express their spin, isospin dependence in terms of nucleon $\boldsymbol{\sigma}$ - and $\boldsymbol{\tau}$-operators demonstrating explicitly the correspondence between these terms and the conventional one-boson exchange potentials.

In order to isolate the simple $q \bar{q}$ exchange terms, sect. 2 gives a brief review of the different types of quark-gluon exchange terms which contribute to the exchange kernels from the coupling of the (3q)-(3q) components to the $(3 q)-(3 q)(q \bar{q})$ components of the two-nucleon wave functions. By comparing the full Wigner transforms, (including the contributions from all 25 types of exchange terms), with the Wigner transforms derived from the simple color-singlet $q \bar{q}$ exchange terms, it is shown that the latter dominate the effective NN potentials in the long-range, $r \geqslant 1.2 \mathrm{fm}$, region and are significant contributors in the $r \sim 1 \mathrm{fm}$ range. Sect. 3 gives the explicit evaluation of the exchange kernels for the simple color-singlet $q \bar{q}$ exchange terms. By expressing their color, spin, iscupin dependence in terms of 
nucleon $\boldsymbol{\sigma}$ - and $\boldsymbol{\tau}$-operators it becomes possible to separate these into spin-independent central, spin-spin, $L S$, and tensor terms. The Wigner transforms of these kernels at $\boldsymbol{P}=0$ are then identified as the simple color-singlet $q \bar{q}$ exchange potentials. In sect. 4 these are compared with the conventional one boson exchange potentials ${ }^{4}$ ), regularized with the usual momentum-cutoff factors ${ }^{5}$ ). Although the $q \bar{q}$ exchange potentials are not in precise quantitative agreement with the one meson exchange potentials, there is a very gratifying correspondence between the various components of the two types of potentials. In particular, the qq exchange potentials corresponding to pseudoscalar meson exchange have no spin-independent central and no $L S$ terms, whereas the potentials corresponding to vector-meson exchange have both spin-spin and spin-independent central terms, as well as $L S$ terms. In addition, the q $\overline{\mathrm{q}}$ exchange potentials and the conventional OBEP's are in good correspondence not only regarding the relative signs and magnitudes of the $\eta, \pi, \omega$ and $\rho$ potentials, and the relative importance of spin-spin and tensor terms, but also regarding their general radial characteristics in the $r \geqslant 1 \mathrm{fm}$ range. One of the significant differences between the two types of potentials involves the steep repulsive rise in the central part of the $\omega$-exchange potential in the $r \leqslant 1 \mathrm{fm}$ range. This is missing in the corresponding $q \bar{q}$ exchange potential although the two types of potentials are in very good quantitative agreement for $r \geqslant 1.2 \mathrm{fm}$. The implications of this finding for the NN interaction are discussed in a concluding section which summarizes results and points to future directions: Specifically, the medium-range attraction carried by a scalar $\sigma$-meson exchange potential in the conventional one-boson exchange picture can be incorporated into our quark model through the $(q \tilde{q})(q \bar{q})$ excitations generated by RPA-type off-shell terms in the Fermi-Breit quark-gluon interaction. The quark exchange kernels needed will be presented in a future study.

\section{Meson exchange diagrams}

The off-shell $(q \bar{q})$-pair creation terms of the Fermi-Breit one-gluon exchange interaction couple the (3q) component of the single-nucleon wave function to $(3 q)(q \bar{q})$ components, with color-singlet $(q \bar{q})$ pairs, only through two types of exchange diagrams. [See e.g. fig. $2 c$ of ref. ${ }^{1}$ ).] In the two-nucleon problem these lead to the NN interaction diagrams shown in fig. 1a. These diagrams have already been singled out by $\mathrm{Yu}$ and $\mathrm{Zhang}^{6}$ ) as the seat of the meson exchange potential in their quark-model study of nucleon-meson vertex functions. In the framework of the resonating group method, with single-nucleon wave functions augmented by $(3 q)(q \bar{q})$ components, such interactions arise from the cross-terms of the exchange kernel, coupling the two-nucleon (3q)-(3q) components to (3q)-(3q)(qव̃) components. The full set of exchange terms follow from the exchange operator [see eq. (48) of ref. $\left.\left.{ }^{1}\right)\right]$ :

$$
\sum_{P} C_{P} P=-3 P_{37}-9 P_{36}+9 P_{36} P_{37}+9 P_{37} P_{36}+18 P_{36} P_{57}+18 P_{36} P_{27},
$$



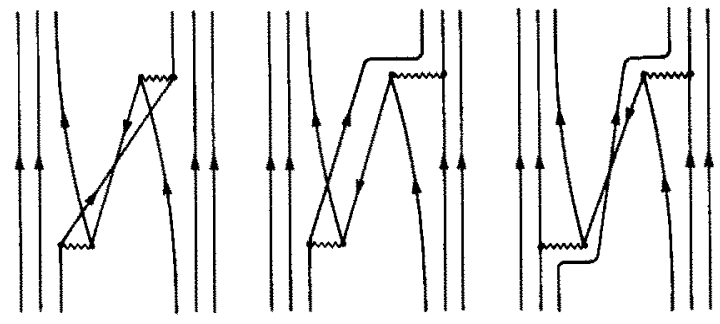

(a)
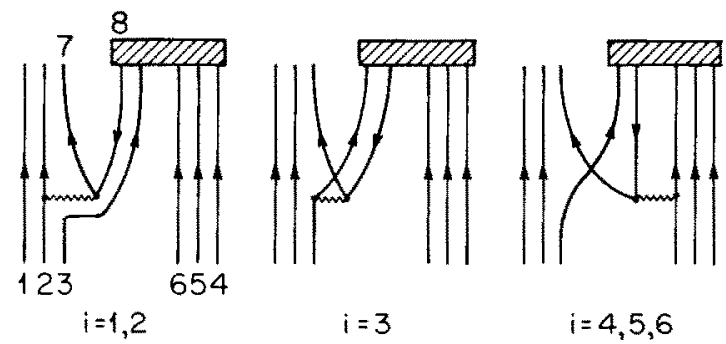

(b) $\mathrm{P}_{37}$ i

Fig. 1. Simple $q \bar{q}$ exchange diagrams.

where the quarks are labeled with indices $i=1,2,3$ in the single-nucleon (3q) cluster, $i=4,5,6$ for the $(3 q)$ cluster and $i=7,8$ for $q, \bar{q}$, respectively, in the $(3 q)(q \bar{q})$ component. It is convenient to introduce diagrams in which the admixed (3q)(qü) components of the single-nucleon wave function are represented by a shaded box. The different exchange types are characterized by the index $i$ which gives the placement of the potential line in the $U_{q \rightarrow q q \bar{q}}\left(\boldsymbol{x}_{i} ; \boldsymbol{x}_{7}, \boldsymbol{x}_{8}\right)$ interaction. Such diagrams for the $P_{37}$ quark exchange terms are shown in fig. $1 \mathrm{~b}$. It is clear that the meson exchanges, via color-singlet q $\bar{q}$ pairs, of fig. 1a result from the combinations of $(i=3)$ with $(i=3)$; $(i=3)$ with $(i=1,2)$; and $(i=1,2)$ with $(i-1,2)$, respectively. Thus, the two types of $P_{37}$ exchange terms with $(i=1,2)$ and $(i=3)$ correspond to simple color-singlet ( $q \bar{q})$ meson exchange terms. The $P_{37}$ exchange terms with $i=4$, 5,6 , on the other hand, involve only a simple quark exchange. Most of the remaining of the 25 possible types of exchange terms lead to quark exchanges and gluon exchanges only. This is illustrated in fig. 2 . The four types of $P_{36}$ exchange terms, also of the "two-cluster" or "semi-direct" variety, with $i=1,2$ or 3 lead only to one-quark and one-gluon exchange; and with $i=4,5$ or 6 to pure one-quark exchange. The $P_{36} P_{37}$ exchange terms with $i=4,5$ or 6 are pure one-quark exchange terms. However, fig. 2 shows that $P_{36} P_{37}$ exchange terms with $i=1,2$ or 3 also lead to color-singlet $\mathrm{q} \overline{\mathrm{q}}$-pair exchanges; but since these are of the complicated "three cluster" variety they must be expected to contribute predominantly to the short-range part of the $\mathrm{NN}$ interaction. The remaining exchange terms of quark exchange 

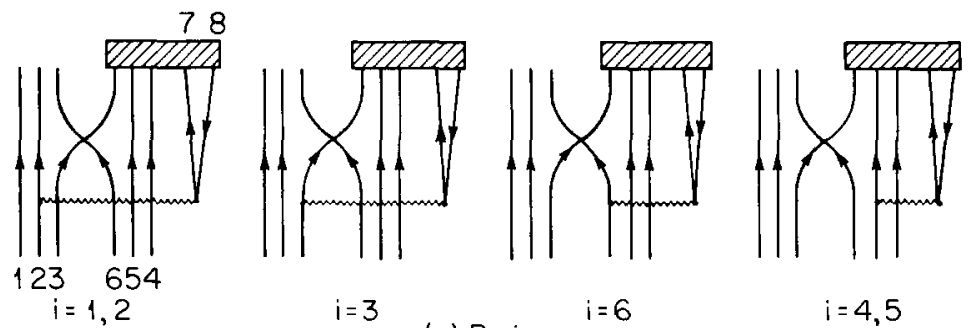

$$
i=3 \quad \text { (a) } P_{36} i
$$

$i=6$

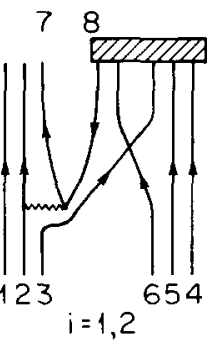

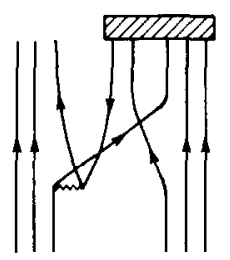

$i=3$

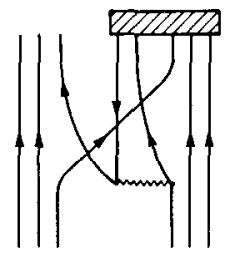

$i=6$

(b) $P_{36} P_{37}{ }^{i}$

Fig. 2. Examples of other quark exchange diagrams.

character, $P_{37} P_{36}, P_{36} P_{57}, P_{36} P_{27}$ (not shown in fig. 2), lead only to pure quark and gluon exchanges or include $q \bar{q}$ exchanges accompanied by additional quark exchanges. The $P_{37}$ exchange terms with $i=1,2$ or 3 have thus been singled out as the seat of meson exchange processes. It is also interesting to note that the $P_{37}$ exchange terms with $i=1,2$ or 3 are the only "semi-direct" terms which give rise to color-singlet $\mathrm{q} \overline{\mathrm{q}}$ exchanges. The remaining "semi-direct" terms, of exchange type

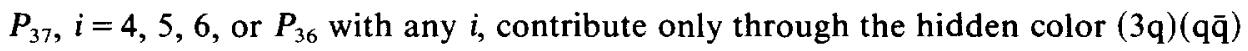
components of the coupling kernel. Henceforth the $P_{37}$ exchange terms with $i=1$, 2, 3 will therefore be denoted as simple color-singlet $q \bar{q}$ exchange terms.

Figs. 3-6 are included to show that these simple color-singlet $q \vec{q}$ exchange terms dominate the effective $\mathrm{NN}$ potentials in the long-range $r \geqslant 1.2 \mathrm{fm}$ domain and are a significant contributor to the potentials in the $r \sim 1 \mathrm{fm}$ range. As in ref. ${ }^{1}$ ), the $\boldsymbol{P}=0$ Wigner transforms of the quark exchange kernels for the cross terms, coupling the (3q)-(3q) component of the two-nucleon wave functions to a specific (3q)$(3 q)(q \bar{q})$ component, with the $(q \bar{q})$ quantum numbers of a real meson, are used as a measure of the effective NN potentials for that "meson". The figures single out the contributions of a single (3q)( $q \bar{q})$ component with color-singlet $(q \bar{q})$ coupled to a (3q) piece with the quantum numbers of the nucleon, $N$. Figs. 3 and 4 show the Wigner transforms for the $\mathrm{N} \eta$ and $\mathrm{N} \pi$ components. For the vector mesons the $\mathrm{N} \omega$ and $\mathrm{N} \rho$ terms gain contributions from two components since the spin $S_{12}$ [in the notation of ref. $\left.{ }^{1}\right)$ ] of the $(3 \mathrm{q})(\mathrm{q} \overline{\mathrm{q}})$ components has the two possible values $S_{12}=\frac{1}{2}$ and $\frac{3}{2}$. Figs. 5 and 6 show the Wigner transforms for $\mathrm{N} \omega$ and $\mathrm{N} \rho$ summed over the two possible $S_{12}$ values. These sums have been chosen partly for simplicity, but also 


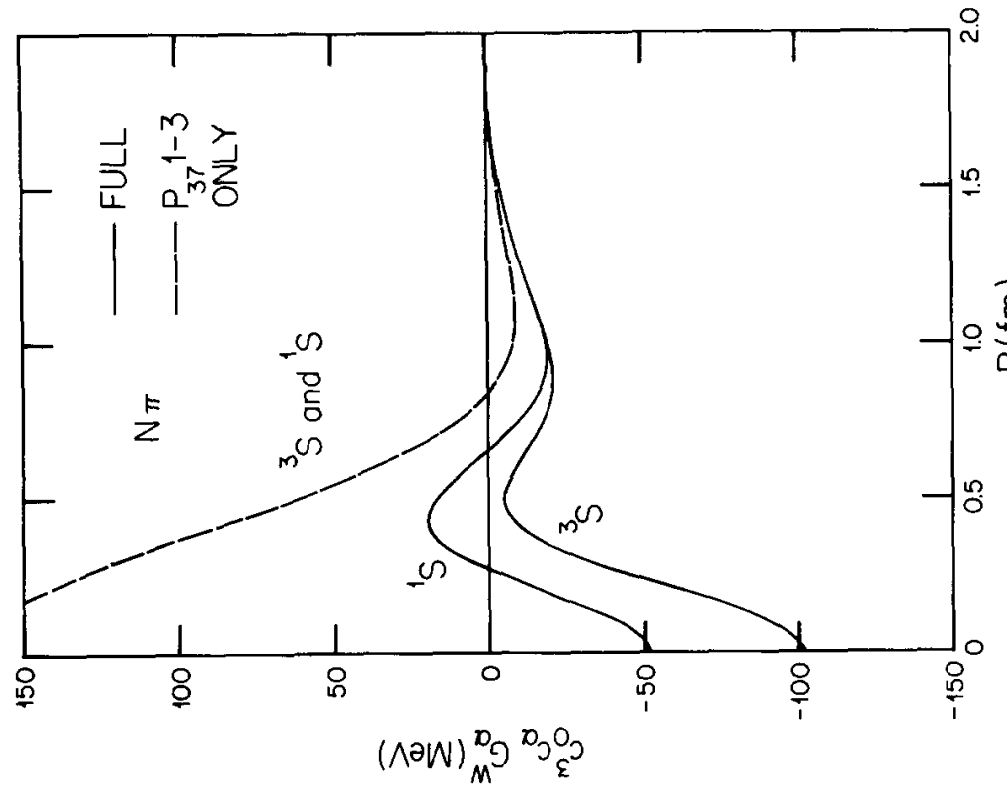

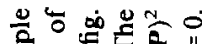
을 की

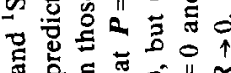
ह त

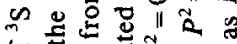
을

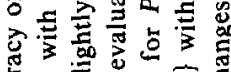

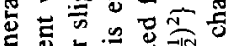
过

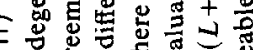
然 드

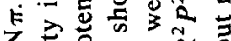

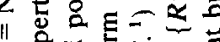

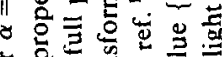
○

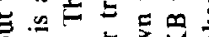

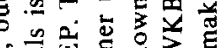
m. 青啠

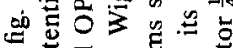

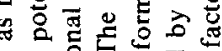
品品焉导

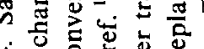

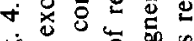

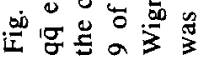

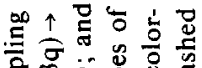

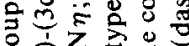

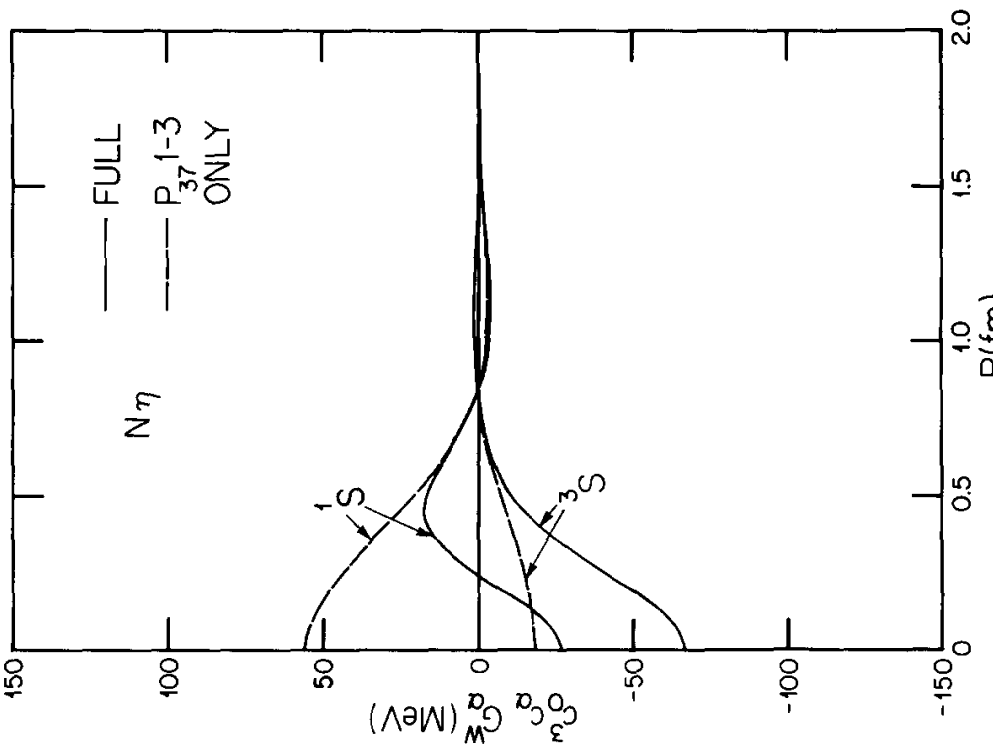

웜 引롤

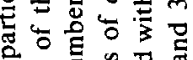

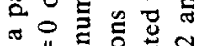

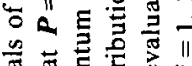
政

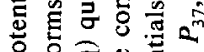

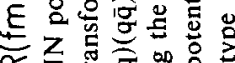
乙 雪

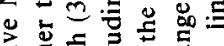
昰高要

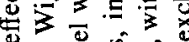

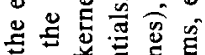
연 드을 을 믕

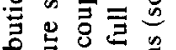

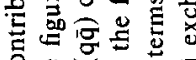

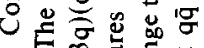
m结票

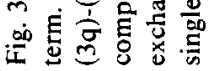



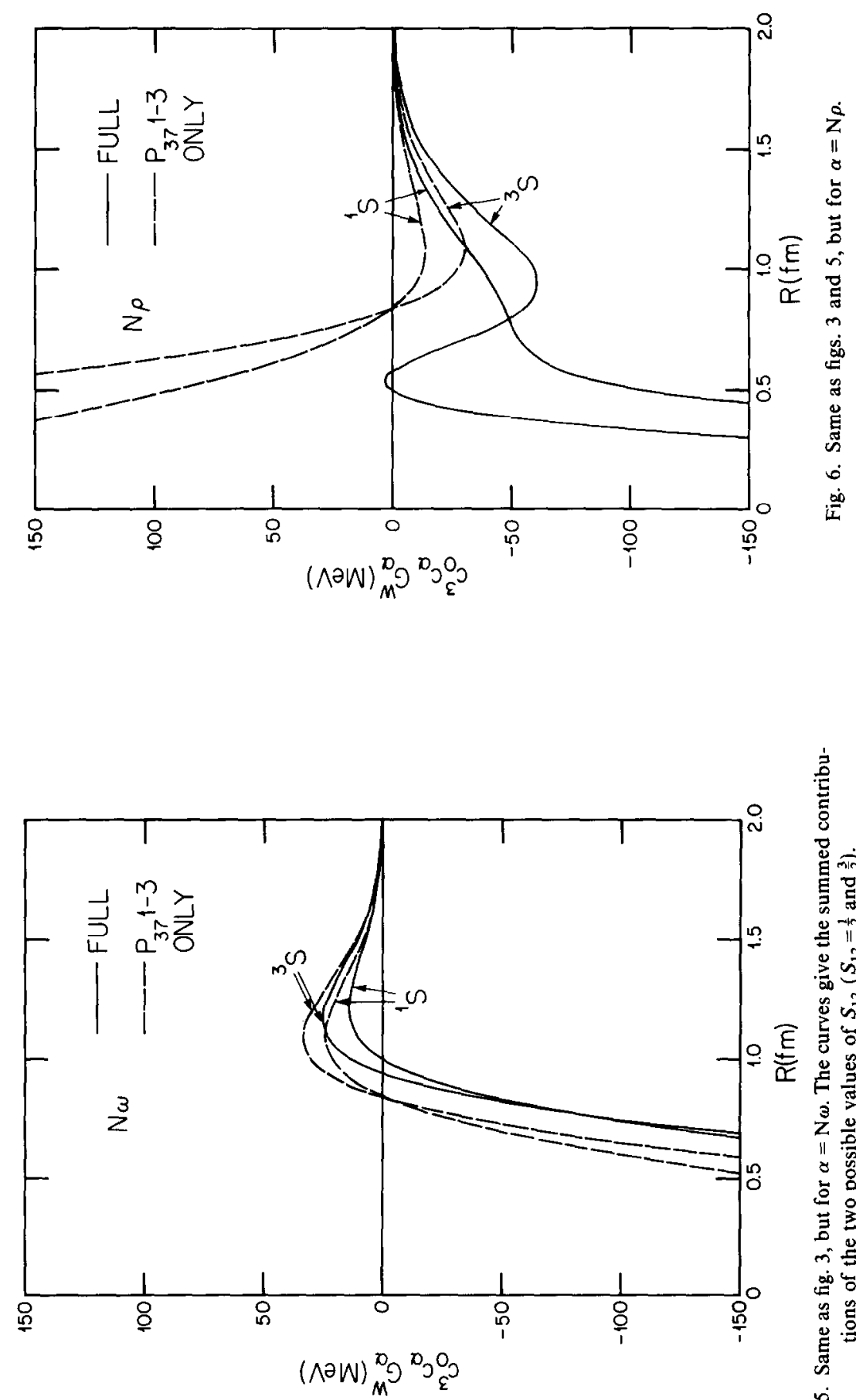
because some terms, such as the $\mathrm{N} \rho S_{12}=\frac{1}{2}$ contributor to the ${ }^{3} \mathrm{~S} N \mathrm{~N}$ potential, are extremely small in the $r \geqslant 1.2 \mathrm{fm}$ range. Note also that $\Delta \pi$ and $\Delta \rho$ potentials are missing. Although these are among the most important contributors to the medium range attractions they gain their strengths from more complicated exchange types. Contributions of $P_{37}$ exchange with $i=1,2,3$ are prohibited for $\Delta \pi$ and $\Delta \rho$ potentials. (For such exchange terms, quark lines 4, 5, 6 of fig. $1 \mathrm{~b}$ must carry the quantum numbers of a nucleon.) Figs. 3-6 compare the full Wigner transforms, including the contributions of all 25 types of exchange terms, with curves for which all but the simple color-singlet $q \bar{q}$ exchange terms have been turned off; that is, for curves which gain their contributions entirely from the $P_{37}$ exchange terms with $i=1,2$ and $i=3$. The importance of the simple color-singlet $q \bar{q}$ exchange terms for the long-range parts of each component of the NN potentials is clearly demonstrated. Finally, the relative simplicity of the simple color-singlet $q \bar{q}$ exchange terms makes it possible to evaluate such exchange kernels, as well as their Wigner transforms, in explicit analytic form.

\section{Simple color-singlet $q \bar{q} \bar{q}$ exchange potentials}

The cross-term of the RGM kernel resulting from the coupling of the (3q)-(3q) component of the two-nucleon wave function to the $(3 q)-(3 q)(q \bar{q})$ components has the form [see ref. $\left.^{1}\right)$,

$$
G\left(\boldsymbol{R}, \boldsymbol{R}^{\prime}\right)=\sum_{\alpha} c_{0}^{3} c_{\alpha} \sum_{P} C_{P} \sum_{O=\mathrm{D}, \mathrm{M}} \sum_{i=1}^{6} G_{P, i}^{O, \alpha}\left(\boldsymbol{R}, \boldsymbol{R}^{\prime}\right),
$$

where

$$
\begin{aligned}
G_{P, i}^{O, \alpha}\left(R, R^{\prime}\right)= & -\frac{4 i}{\pi} \alpha_{s} \hbar c\left\langle P\left[\phi_{\frac{11}{22}}(3 q) \tilde{\phi}_{\alpha, \frac{12}{22}}((3 q)(q \bar{q}))\right]_{S M_{S} T M_{T}} \delta\left(r_{01}-R\right)\right| \\
& \times \delta\left(x_{7}-x_{8}\right) F^{O}\left(x_{i}, x_{7}\right) \cdot \mathscr{P}^{O}(i ; 7,8) \\
& \times\left|\left[\phi_{\frac{11}{22}}(3 q) \phi_{\frac{11}{22}}(3 q)\right]_{S^{\prime} M_{S}^{\prime} T M_{T}} \delta\left(r_{00}-R^{\prime}\right)\right\rangle,
\end{aligned}
$$

and where the $(3 q)(q \bar{q})$ component of the single-nucleon internal wave function, characterized by quantum numbers $\alpha$, is given by

$$
\tilde{\phi}_{\alpha, S M_{S} T M_{T}}((3 q)(\mathrm{q} \overline{\mathrm{q}}))=i N_{\alpha \alpha}^{-1 / 2}\left[\chi_{l=1}^{\text {h.o. }}(\rho)\left[\phi_{S_{2} T_{2}(\lambda \lambda)}(3 \mathrm{q}) \phi_{S_{3} T_{3}(\lambda \lambda)}(\mathrm{q} \overline{\mathrm{q}})\right]_{S_{23} T(00)}\right]_{S M_{S} T M_{T}} \text {. }
$$

The square brackets denote spin, isospin, and color coupling. Note that the quantum number $S_{23}$, part of $\alpha$, was previously named $S_{12}$, see ref. ${ }^{1}$ ). In eq. (2) $c_{0}$ and $c_{\alpha}$ are the amplitudes of the $\phi(3 q)$ and $\phi_{\alpha}((3 q)(q \bar{q}))$ components of the single-nucleon internal function. As in ref. $\left.{ }^{1}\right)$, only terms linear in $c_{\alpha}$ have been retained. In the present investigation the exchange types, sums over $P$ and $i$, will be restricted to the simple $\mathrm{q} \overline{\mathrm{q}}$ mesonic exchange type, i.e. $P=P_{37}$ and $i=1,2$ or 3 only [see eq. (1) 
and fig. 1]; and consequently the states $\alpha$ are restricted to the color-singlet variety, $(\lambda \lambda)=(00)$, with $S_{2} T_{2}=\frac{11}{22}$, the quantum numbers of the nucleon, i.e. only the contributions of states $\alpha=\mathrm{N} \eta, \mathrm{N} \pi$, and $\mathrm{N} \omega, \mathrm{N} \rho$ with $S_{23}=\frac{1}{2}, \frac{3}{2}$, need to be considered for this simple exchange type. In eq. (3) the q $\bar{q}$-pair creation interaction $U_{\mathrm{q} \rightarrow \mathrm{qq} \bar{q}}$ has been given in vector form. The spin-vector operators $\mathscr{P}^{\circ}(i ; 7,8)$ give the spin, isospin, color factors of the interaction, see specifically eqs. (8c,d) of ref. '). The spatial vector factors $\boldsymbol{F}^{\mathscr{O}}\left(\boldsymbol{x}_{\mathrm{i}}, \boldsymbol{x}_{7}\right)$ are of two types, $\mathcal{O}=\mathrm{D}$ (derivative type) and $\mathscr{O}=\mathrm{M}$ (momentum type):

$$
F^{\mathrm{D}}\left(\boldsymbol{x}_{\mathrm{i}}, \boldsymbol{x}_{7}\right)=\frac{\partial}{\partial \boldsymbol{r}} F(\boldsymbol{r}) ; \quad F^{\mathrm{M}}\left(\boldsymbol{x}_{\mathrm{i}}, \boldsymbol{x}_{7}\right)=F(\boldsymbol{r}) \frac{\partial}{\partial \boldsymbol{x}_{\mathrm{i}}},
$$

with

$$
F(r)=\frac{\pi}{2} \frac{\hbar}{m c} \frac{1}{r}=\frac{\pi}{2} x \frac{b}{r} ; \quad r=\left|x_{\mathrm{i}}-x_{7}\right|, \quad x=\frac{\hbar}{m c} \frac{1}{b} .
$$

In eq. (3), $r_{0 \alpha}$ give the relative position vectors $\left(r_{00}\right.$ from the c.m. of one (3q) cluster to the other, and $\boldsymbol{r}_{01}$ from the c.m. of the $(3 q)(q \bar{q})$ cluster to the c.m. of the other (3q) cluster), while $\rho$ is the relative position vector from the c.m. of the (3q) cluster to the c.m. of the ( $(\bar{q} \bar{q})$ cluster. As in ref. $\left.{ }^{1}\right)$, the ( $\left.3 q\right)-(q \bar{q})$ relative-motion function is approximated by a harmonic-oscillator function for the energetically lowest p-wave. For the separation of the kernel into space and spin, isospin, color parts it is convenient to express these p-wave oscillator functions in terms of a vector coherent state:

$$
\chi^{\boldsymbol{e}}(\boldsymbol{\rho}, \gamma)=2 \sqrt{\gamma}(\boldsymbol{e} \cdot \boldsymbol{\rho})\left[\frac{2 \gamma}{\pi}\right]^{3 / 4} \mathrm{e}^{-\gamma \rho^{2}}=\boldsymbol{e} \cdot\left(\frac{\partial}{\partial \boldsymbol{e}} \boldsymbol{A}_{\gamma}(\boldsymbol{\rho}, \boldsymbol{e})\right)_{\boldsymbol{e}=0},
$$

with

$$
A_{\gamma}(\boldsymbol{\rho}, \boldsymbol{e})=\left[\frac{2 \gamma}{\pi}\right]^{3 / 4} \exp \left[-\gamma\left(\boldsymbol{\rho}-\frac{e}{\sqrt{\gamma}}\right)^{2}+\frac{1}{2} e^{2}\right],
$$

where a specific $0 p$ oscillator function is regained through

$$
\chi_{1 \mu}^{\text {h.o. }}(\boldsymbol{\rho}, \gamma)=\frac{3}{4 \pi} \int \mathrm{d} \hat{\mathbf{e}} e_{\mu} \chi^{e}(\boldsymbol{\rho}, \gamma)
$$

and where $e$ is a real unit vector with standard spherical components $e_{\mu}\left(\equiv e_{1 \mu}\right)$. In terms of such functions the exchange kernels of eq. (3) can be written as

$$
G_{P, i}^{O, \alpha}\left(\boldsymbol{R}, \boldsymbol{R}^{\prime}\right)=-\frac{4}{\pi} \alpha_{\mathrm{s}} \hbar c N_{\alpha \alpha}^{-1 / 2} \frac{3}{4 \pi} \int \mathrm{d} \hat{\boldsymbol{e}}\left(\boldsymbol{I}_{P, i}^{O, e}\left(\boldsymbol{R}, \boldsymbol{R}^{\prime}\right) \cdot \boldsymbol{X}_{P, i}^{O, \alpha ; e}\right),
$$

where the space part is expressed in terms of the 0s internal orbital functions by

$$
\begin{aligned}
\boldsymbol{I}_{P, i}^{\sigma, e}\left(\boldsymbol{R}, \boldsymbol{R}^{\prime}\right)= & \left\langle\boldsymbol{P} \phi_{0 \mathrm{~s}}(3 \mathrm{q}) \phi_{0 \mathrm{~s}}(3 \mathrm{q}) \phi_{0 \mathrm{~s}}(\mathrm{q} \overline{\mathrm{q}}) \chi^{e}(\boldsymbol{\rho}, \gamma) \delta\left(\boldsymbol{r}_{01}-\boldsymbol{R}\right)\right| \\
& \times \delta\left(\boldsymbol{x}_{7}-\boldsymbol{x}_{8}\right) \boldsymbol{F}^{\circ}\left(\boldsymbol{x}_{i}, \boldsymbol{x}_{7}\right)\left|\phi_{0 \mathrm{~s}}(3 \mathrm{q}) \phi_{0 \mathrm{~s}}(3 \mathrm{q}) \delta\left(\boldsymbol{r}_{00}-\boldsymbol{R}^{\prime}\right)\right\rangle .
\end{aligned}
$$


This space part of the RGM kernel is evaluated through its Bargmann transform or complex generator-coordinate kernel $^{7,8}$ )

$$
\boldsymbol{I}_{P, i}^{O, e}\left(\boldsymbol{R}, \boldsymbol{R}^{\prime}\right)=\int \mathrm{d} \mu(z) \mathrm{d} \mu\left(z^{\prime}\right) A_{\gamma_{1}}(\boldsymbol{R}, z) A_{\gamma_{0}}^{*}\left(\boldsymbol{R}^{\prime}, z^{\prime}\right) \boldsymbol{I}_{P, i}^{O, e G C M}\left(z, z^{\prime}\right)
$$

where the $z, z^{\prime}$ are 3-dimensional Bargmann space variables, and the 6-dimensional $\mathrm{d} \mu(z)$ include the Bargmann weighting function $\pi^{-3} \exp \left(-z \cdot z^{*}\right)$. The complex GCM kernel is given by the term linear in $e$ :

$$
\begin{aligned}
\left(I_{P, i}^{\mathcal{E}, \mathrm{GCM}}\left(z, z^{\prime}\right)\right)_{\mu}= & \boldsymbol{e} \cdot\left(\frac{\partial}{\partial \boldsymbol{e}}\left\langle P A_{\gamma_{1}}\left(\boldsymbol{r}_{01}, z\right) A_{\gamma}(\boldsymbol{\rho}, \boldsymbol{e}) \phi_{0 \mathrm{~s}}(3 \mathrm{q}) \phi_{0 \mathrm{~s}}(3 \mathrm{q}) \phi_{0 \mathrm{~s}}(\mathrm{q} \tilde{\mathrm{q}})\right|\right. \\
& \left.\times \delta\left(\boldsymbol{x}_{7}-\boldsymbol{x}_{8}\right) F_{\mu}^{\mathcal{G}}\left(\boldsymbol{x}_{i}, \boldsymbol{x}_{7}\right)\left|\boldsymbol{A}_{\gamma_{0}}\left(\boldsymbol{r}_{00}, z^{\prime}\right) \phi_{0 \mathrm{~s}}(3 q) \phi_{0 \mathrm{~s}}(3 \mathrm{q})\right\rangle\right)_{\boldsymbol{e}=0}
\end{aligned}
$$

The three range parameters $\left[\gamma_{1}=15 / 16 b^{2}, \gamma=3 / 5 b^{2}, \gamma_{0}=3 / 4 b^{2}\right]$ differ in their reduced mass factors; $b$ is the standard oscillator length parameter. These GCM kernels are easiest to evaluate if the scalar function, $F(r)$ of eq. (5), is expanded in terms of gaussians exp $\left(-\chi^{2} r^{2}\right)$. In particular,

$$
\frac{b}{r}=\frac{2 b}{\sqrt{\pi}} \int_{0}^{\infty} \mathrm{d} \chi \mathrm{e}^{-\chi^{2} r^{2}}
$$

Note also that the derivative operations of eq. (5) commute with the $x$-integration. The GCM kernels for the above vector operators can be expressed in terms of cartesian $j k$ components,

$$
\left(I_{P, i}^{O, e G C M}\left(z, z^{\prime}\right)\right)_{j}=\sum_{k}\left(I_{P, i}^{O C G M}\left(z, z^{\prime}\right)\right)_{j k} e_{k} .
$$

With the replacement, $b / r \rightarrow \exp \left(-\chi^{2} r^{2}\right)$, these have the simple form

$$
I_{j k}^{\mathrm{GCM}}\left(z, z^{\prime}\right)=\left\{\left(a z^{*}+b z^{\prime}\right)_{j}\left(c z^{*}+d z^{\prime}\right)_{k}+e \delta_{j k}\right\} \exp \left[-\frac{1}{2} f z^{* 2}-\frac{1}{2} g z^{\prime 2}+h z^{*} \cdot z^{\prime}\right],
$$

where the coefficients, $a, \ldots, h$, have been evaluated for the different exchange terms, $P, i$, and the two $\mathcal{O}$ by the techniques of refs. ${ }^{3,7,8}$ ).

The spin, isospin, color parts needed to construct the full kernels, through eq. (7), are given by

$$
\boldsymbol{X}_{P, i}^{O, \alpha ; e}=\left\langle P\left[\xi_{112}\left[e_{1} \xi_{\alpha, S_{23} T=\frac{1}{2}}\right]_{\frac{11}{22}}\right]_{S M_{S} T M_{T}}\left|\mathscr{P}^{\mathcal{O}}(i ; 7,8)\right|\left[\xi_{\frac{11}{22}} \xi_{\frac{11}{22}}\right]_{S^{\prime} M_{S}^{\prime} T M_{T}}\right\rangle,
$$

where $\xi_{\frac{11}{22}}$ and $\xi_{\alpha, S_{23} T=\frac{1}{2}}$ are the spin, isospin, color parts of the color singlet $\phi(3 q)$ and $\phi_{\alpha}((3 q)(q \bar{q}))$, respectively. Eq. (14) involves the angular momentum coupling of the $l=1$ vector operator $e$ with the spin $S_{23}$ to resultant angular momentum $\frac{1}{2}$. By a recoupling transformation to the form $\left[e_{1}\left[\xi_{22} \xi_{\alpha, S_{232}}\right]_{S_{123} T}\right]_{S M_{S} T M_{T}}$ and explicit 
expansion, this can be put in the form

$$
\begin{aligned}
& \left(X_{P, i}^{O, \alpha ; e}\right)_{\mu}=e_{M_{S}-M_{S^{-}}^{\prime}}^{*} \sum_{S_{123}}\left\langle 1\left(M_{S}-M_{S}^{\prime}-\mu\right) S_{123}\left(M_{S}^{\prime}+\mu\right) \mid S M_{S}\right\rangle \\
& \times\left\langle S^{\prime} M_{S}^{\prime} 1 \mu \mid S_{123}\left(M_{S}^{\prime}+\mu\right)\right\rangle(-1)^{S_{123}+S_{23}+S+\frac{3}{2}} U\left(1 S_{23} S_{2}^{1} ; \frac{1}{2} S_{123}\right) \\
& \times\left\langle S_{123}\left\|X_{P, i}^{O, \alpha}\right\| S^{\prime}\right\rangle,
\end{aligned}
$$

where the reduced matrix element,

$$
\left\langle S_{123}\left\|X_{P, i}^{O, \alpha}\right\| S^{\prime}\right\rangle \equiv\left\langle P\left[\xi_{\frac{11}{22}} \xi_{\alpha, S_{23} \frac{1}{2}}\right]_{S_{123} T}\left\|\mathscr{S}^{\sigma}(i ; 7,8)\right\|\left[\xi_{\frac{11}{22}} \xi_{\frac{11}{22}}\right]_{S^{\prime} T}\right\rangle,
$$

is given by the defining relation [(full matrix element $)=($ reduced matrix element $) \times$ (Wigner coefficient)] without dimensional factors such as $\left(2 S_{123}+1\right)^{-1 / 2}$. To express the vector operators $\boldsymbol{X}_{P, i}^{\sigma, \alpha}, e$ of eqs. (14), (15) in terms of the vector $\boldsymbol{e}$ and nucleon operators $\boldsymbol{\sigma}_{1}, \boldsymbol{\sigma}_{2}$, it is convenient to subject these to one more recoupling transformation to yield

$$
\begin{aligned}
\left(X_{P, i}^{O, \alpha ; e}\right)_{\mu}= & \sum_{S_{123}} \sum_{f m_{f}} \sum_{\nu} e_{\nu}^{*}\left\langle 1 \mu 1 \nu \mid f m_{f}\right\rangle\left\langle S^{\prime} M_{S}^{\prime} f m_{f} \mid S M_{S}\right\rangle U\left(S^{\prime} 1 S 1 ; S_{123} f\right) \\
& \times(-1)^{\frac{1}{2}+S_{23}} U\left(1 S_{23} S \frac{1}{2} ; \frac{1}{2} S_{123}\right)\left\langle S_{123}\left\|X_{P, i}^{O, \alpha}\right\| S^{\prime}\right\rangle
\end{aligned}
$$

Here, the dependence on two-nucleon spins, through the $\left\langle S^{\prime} M_{S}^{\prime} f m_{f} \mid S M_{S}\right\rangle$ factor, can be represented in terms of nucleon spin operators, with ranks $f=0,1,2$, through

$$
S_{m_{f}}^{f}=\left\{\begin{array}{l}
1 \\
\frac{1}{2}\left(\boldsymbol{\sigma}_{1}+\sigma_{2}\right)_{m_{f}} \\
{\left[\boldsymbol{\sigma}_{1} \sigma_{2}\right]_{m_{f}}^{2}}
\end{array} \quad \text { for } f=\left\{\begin{array}{l}
0 \\
1 \\
2,
\end{array}\right.\right.
$$

where we use the two-nucleon matrix elements

$$
\left\langle\left[\chi_{\frac{11}{22}}(\mathrm{~N}) \chi_{\frac{11}{22}}(\mathrm{~N})\right]_{S M_{S} T M_{T}}\left|S_{m_{f}}^{f}\right|\left[\chi_{\frac{11}{2}}(\mathrm{~N}) \chi_{\frac{11}{22}}(\mathrm{~N})\right]_{S^{\prime} M_{S}^{\prime} T M_{\gamma}}\right\rangle=\left\langle S^{\prime} M_{S}^{\prime} f m_{f} \mid S M_{S}\right\rangle\left\langle S\left\|S^{f}\right\| S^{\prime}\right\rangle
$$

to fix the coefficients. For NN scattering, with isospin-conserving interaction, the combination of the Pauli principle and parity conservation requires $S^{\prime}=S$, so that the two-nucleon reduced matrix elements have the simple form

$$
\left\langle S\left\|S^{f}\right\| S^{\prime}\right\rangle=\delta_{S S^{\prime}}\left\{\delta_{f 0}+\delta_{S 1}\left(\sqrt{2} \delta_{f 1}+2\left[\frac{5}{3}\right]^{1 / 2} \delta_{f 2}\right)\right\} .
$$

The vector operators $\boldsymbol{X}_{P, i}^{\sigma, \alpha ; e}$ can thus be expressed in terms of nucleon vector uperators $\tilde{\boldsymbol{X}}_{P, i}^{\sigma, \alpha}, e$, with two-nucleon matrix elements

$$
\boldsymbol{X}_{P, i}^{O, \alpha ; e}=\left\langle\left[\chi_{\frac{11}{22}}(\mathrm{~N}) \chi_{\frac{11}{22}}(\mathrm{~N})\right]_{S M_{S} T M_{T}}\left|\tilde{\boldsymbol{X}}_{P, i}^{O, \alpha ; e}\right|\left[\chi_{\frac{11}{22}}(\mathrm{~N}) \chi_{\frac{11}{22}}(\mathrm{~N})\right]_{S M_{S}^{\prime} T M_{T}}\right\rangle,
$$

where (with $\hat{f} \equiv[2 f+1]^{1 / 2}$ )

$$
\begin{aligned}
& \left(\tilde{X}_{P, i}^{O, \alpha ; e}\right)_{\mu}=\sum_{S_{123}} \sum_{f}\left[e_{1} S^{f}\right]_{\mu}^{1} \frac{\hat{f}(-1)^{\frac{3}{2}+S_{23}}}{\sqrt{3}\left\langle S\left\|S^{f}\right\| S\right\rangle} U\left(S 1 S 1 ; S_{123} f\right) U\left(1 S_{23} S_{2}^{1} ; \frac{1}{2} S_{123}\right) \\
& \times\left\langle S_{123}\left\|\chi \chi_{P, i}^{\sigma, \alpha}\right\| S\right\rangle \text {. }
\end{aligned}
$$


By expanding $\left[e_{1} S^{f}\right]_{\mu}^{1}$, using identities such as $\left[a_{1} b_{1}\right]_{\mu}^{1}=\sqrt{\frac{1}{2}} i[a \times b]_{\mu}$, where $[a \times b]$ is the ordinary vector product, the two-nucleon vector operators can be put in the form

$$
\begin{aligned}
\tilde{\boldsymbol{X}}_{P, i}^{\sigma, \alpha \cdot e}= & \sum_{S_{123}}\left\{-e_{3}^{\frac{1}{3}} \hat{S}_{123} / \hat{S}+\delta_{S_{1} \frac{1}{8} \sqrt{\frac{1}{3}}} \hat{S}_{123} i[e \times S]\left[4-S_{123}\left(S_{123}+1\right)\right]\right. \\
& +\delta_{S 1}\left[\frac{1}{2} \boldsymbol{\sigma}_{1}\left(e \cdot \sigma_{2}\right)+\frac{1}{2} \sigma_{2}\left(e \cdot \sigma_{1}\right)-\frac{1}{3} e\left(\sigma_{1} \cdot \sigma_{2}\right)\right] \frac{1}{40} \sqrt{\frac{1}{3}} \hat{S}_{123} \\
& \left.\times\left(3\left[4-S_{123}\left(S_{123}+1\right)\right]\left[3-S_{123}\left(S_{123}+1\right)\right]-16\right)\right\} \\
& \times(-1)^{S_{123}+S_{23}+S+\frac{3}{2}} U\left(1 S_{23} S_{2}^{1} ; \frac{1}{2} S_{123}\right)\left\langle S_{123}\left\|X_{P, i}^{O, \alpha}\right\| S\right\rangle,
\end{aligned}
$$

where $\boldsymbol{S}=\frac{1}{2}\left(\boldsymbol{\sigma}_{1}+\boldsymbol{\sigma}_{2}\right)$.

Using the RGM analog of eq. (12)

$$
\left(I_{P, i}^{O, e}\left(\boldsymbol{R}, \boldsymbol{R}^{\prime}\right)\right)_{j}=\sum_{k}\left(I_{P, i}^{\sigma}\left(\boldsymbol{R}, \boldsymbol{R}^{\prime}\right)\right)_{j k} e_{k},
$$

and the simple relation

$$
\frac{3}{4 \pi} \int \mathrm{d} \hat{\boldsymbol{e}} e_{j} e_{k}=\delta_{j k}
$$

for cartesian components of $e$, the space and spin, isospin, color factors of the kernels can now be combined via the relation (cf. eq. (7))

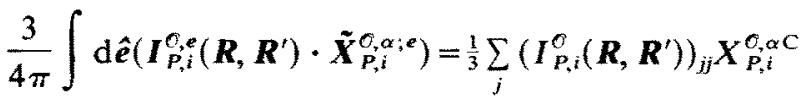

$$
\begin{aligned}
& +i \sum_{j k l} \varepsilon_{j k l}\left(I_{P, i}^{\sigma}\left(\boldsymbol{R}, \boldsymbol{R}^{\prime}\right)\right)_{j k} \boldsymbol{S}_{l} \boldsymbol{X}_{P, 2}^{\sigma_{p}, \alpha S} \\
& +\left[\sum_{j k}\left(\frac{1}{2}\left(I_{P, i}^{\odot}\left(\boldsymbol{R}, \boldsymbol{R}^{\prime}\right)\right)_{j k}+\frac{1}{2}\left(I_{P, i}^{\odot}\left(\boldsymbol{R}, \boldsymbol{R}^{\prime}\right)\right)_{k j}\right) \boldsymbol{\sigma}_{1 j} \boldsymbol{\sigma}_{2 k}\right. \\
& \left.-\frac{1}{3} \sum_{j}\left(I_{P, i}^{\sigma}\left(\boldsymbol{R}, \boldsymbol{R}^{\prime}\right)\right)_{j}\left(\boldsymbol{\sigma}_{1} \cdot \boldsymbol{\sigma}_{2}\right)\right] X_{P, i}^{\sigma, \alpha} \\
& \equiv \sum_{\Omega=\mathrm{C}, L S, \mathrm{~T}} \mathcal{M}_{P, i}^{O, \Omega}\left(\boldsymbol{R}, \boldsymbol{R}^{\prime}\right) X_{P, i}^{O, \alpha \Omega} .
\end{aligned}
$$

The three terms of this expression will lead to the central (C), spin-orbit ( $L S$ ) and tensor $(T)$ parts of the potentials. The three types of spin, isospin, color factors are given by

$$
\begin{aligned}
& X_{P, i}^{\sigma_{,}, \alpha} \equiv \sum_{S_{123}}(-1)^{S_{123}+S_{23}+S+\frac{3}{2}} U\left(1 S_{23} S_{2}^{1} ; \frac{1}{2} S_{123}\right)\left\langle S_{123}\left\|X_{P, i}^{O, \alpha}\right\| S\right\rangle, \\
& \times\left\{\begin{array} { l } 
{ - \hat { S } _ { 1 2 3 } / \hat { S } } \\
{ \delta _ { S 1 \frac { 1 } { 8 } \sqrt { \frac { 1 } { 3 } } } \hat { S } _ { 1 2 3 } [ 4 - S _ { 1 2 3 } ( S _ { 1 2 3 } + 1 ) ] } \\
{ \delta _ { S 1 } \frac { 1 } { 4 0 } \sqrt { \frac { 1 } { 3 } } \hat { S } _ { 1 2 3 } \{ 3 [ 4 - S _ { 1 2 3 } ( S _ { 1 2 3 } + 1 ) ] [ 3 - S _ { 1 2 3 } ( S _ { 1 2 3 } + 1 ) ] - 1 6 \} }
\end{array} \quad \text { for } \left\{\begin{array}{l}
\Omega=\mathrm{C} \\
\Omega=L S \\
\Omega=\mathrm{T} .
\end{array}\right.\right.
\end{aligned}
$$


For the construction of the exchange kernels an explicit evaluation of these spin, isospin, color factors is needed. For the special case of the color singlet $\mathrm{q} \overline{\mathrm{q}}$ exchanges, with exchange type restricted to $P=P_{37}, \quad i=1,2,3$, and with $\alpha=$ $\left[S_{2} T_{2}(\lambda \lambda) S_{3} T_{3}(\lambda \lambda)\right] S_{23} T_{23}=\frac{1}{2}$ restricted to color singlet $(\lambda \lambda)=(00)$ and nucleon (3q) type, $S_{2} T_{2}=\frac{11}{22}$, these can be expressed in terms of single-nucleon spin, isospin, color factors; and their dependence on two-nucleon $S$ and $T$ quantum numbers can be expressed in terms of nucleon $\sigma$ - and $\tau$-operators. Recoupling transformations of the type used in ref. ${ }^{1}$ ) lead to

$$
X_{P_{37}, i}^{C_{3} \alpha}=\delta_{\alpha,\left[122(00) S_{3} T_{3}(00)\right] S_{23:}:} \sum_{S_{13}} Y_{S_{23} S_{13}}^{\Omega}\left(S_{3} T_{3} ; S T\right) \sqrt{\frac{1}{2}} \hat{S}_{13} X_{(34), 1}^{C_{1} \alpha_{0}}
$$

with $\alpha_{0}=\left[\frac{11}{22}(00) S_{3} T_{3}(00)\right] S_{13 \frac{1}{2}}$, and with

$$
\begin{aligned}
& Y^{\Omega}\left(S_{3} T_{3} ; S T\right)=\left[\delta_{T_{3} 0}+\delta_{T_{3} 1 \frac{1}{3}}\left(\tau_{1} \cdot \tau_{2}\right)\right]
\end{aligned}
$$

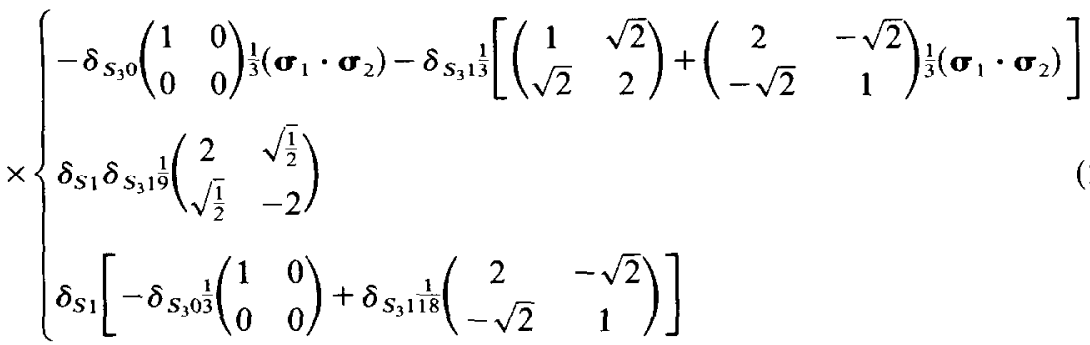

for $\Omega=\mathrm{C}, L S, \mathrm{~T}$, respectively, where we use the matrix notation

$$
Y^{\Omega}=\left(Y_{S_{23} S_{13}}^{\Omega}\right)=\left(\begin{array}{cc}
Y_{11}^{\Omega} & Y_{\frac{13}{22}}^{\Omega} \\
Y_{\frac{13}{31}}^{\Omega} & Y_{\frac{13}{32}}^{\Omega}
\end{array}\right) .
$$

In eq. (28a), the $X_{(34), i}^{C, \alpha_{0}}$ are the single-nucleon spin, isospin, color factors which are tabulated in table 1 a of ref. ${ }^{1}$ ).

In order to convert the exchange kernels into effective NN potentials, it is still necessary to evaluate the Wigner transforms of the above RGM kernels. For the

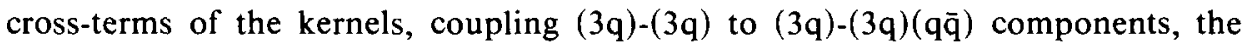
Wigner transforms of the symmetrized RGM kernels are needed,

$$
G^{\mathscr{S}}\left(\boldsymbol{R}, \boldsymbol{R}^{\prime}\right)=G\left(\boldsymbol{R}, \boldsymbol{R}^{\prime}\right)+G\left(\boldsymbol{R}^{\prime}, \boldsymbol{R}\right)^{\dagger},
$$

since the $(3 q)(q \bar{q})$ admixtures in the single-nucleon internal functions appear symmetrically in both bra and ket. The needed Wigner transforms for the full kernels are then

$$
G_{\mathrm{W}}(\boldsymbol{R}, \boldsymbol{P})=\int \mathrm{d} s \exp \left[\frac{i}{\hbar}(\boldsymbol{s} \cdot \boldsymbol{P})\right] G^{\mathscr{P}}\left(\boldsymbol{R}-\frac{1}{2} s, \boldsymbol{R}+\frac{1}{2} s\right),
$$

where this particular form of the Wigner transform applies for the case when the coefficient of the $\left(\boldsymbol{R} \cdot \boldsymbol{R}^{\prime}\right)$ term in the exponential factor of the kernel is positive, 
$h>0$. [See ref. ${ }^{1}$ ), and eq. (13) for the definition of $h$; and note that only $h>0$ occurs for terms of exchange type $P=P_{37}, i=1,2,3$.] It is convenient to define the analogous Wigner transforms for the corresponding symmetrized forms of the orbital kernels $\mathcal{M}_{P, i}^{O, \Omega}\left(\boldsymbol{R}, \boldsymbol{R}^{\prime}\right)$, defined through the last line of eq. (26):

$$
\hat{G}_{P, l_{\mathrm{w}}}^{O, \Omega}(\boldsymbol{R}, \boldsymbol{P})=\int \mathrm{d} s \exp \left[\frac{i}{\hbar}(\boldsymbol{s} \cdot \boldsymbol{P})\right] \mathcal{M}_{P, i}^{\mathscr{S O}, \Omega}\left(\boldsymbol{R}-\frac{1}{2} s, \boldsymbol{R}+\frac{1}{2} s\right) .
$$

For the simple $\mathrm{q} \overline{\mathrm{q}}$ mesonic exchange terms, with $P=P_{37}$ and $i=1,2,3$, these are particularly simple and can be given in explicit analytic form. In a simplified shorthand notation, in which the indices $O, P, i$ are omitted,

where

$$
\hat{G}_{\mathrm{W}}^{\Omega}(\boldsymbol{R}, \boldsymbol{P})=\left\{\begin{array}{l}
\mathscr{G}_{\mathrm{W}}^{\mathrm{C}}(\boldsymbol{R}, \boldsymbol{P}) \\
\mathscr{G}_{\mathrm{W}}^{L S}(\boldsymbol{R}, \boldsymbol{P})(\boldsymbol{L} \cdot \boldsymbol{S}) \\
\mathscr{G}_{\mathrm{W}}^{\mathrm{T}}(\boldsymbol{R}, \boldsymbol{P}) \boldsymbol{S}_{12}+\mathscr{G}_{\mathrm{W}}^{\mathrm{T}}(\boldsymbol{R}, \boldsymbol{P}) \boldsymbol{S}_{12}^{\prime}+\mathscr{G}_{\mathrm{W}}^{\mathrm{T}}(\boldsymbol{R}, \boldsymbol{P}) S_{12}^{\prime \prime},
\end{array}\right.
$$

$$
\begin{gathered}
\boldsymbol{L}=\frac{1}{\hbar}[\boldsymbol{R} \times \boldsymbol{P}], \quad \boldsymbol{S}=\frac{1}{2}\left(\boldsymbol{\sigma}_{1}+\boldsymbol{\sigma}_{2}\right), \\
S_{12}=3\left(\sigma_{1} \cdot \hat{\boldsymbol{R}}\right)\left(\boldsymbol{\sigma}_{2} \cdot \hat{\boldsymbol{R}}\right)-\left(\sigma_{1} \cdot \sigma_{2}\right), \\
S_{12}^{\prime}=3\left(\sigma_{1} \cdot \hat{\boldsymbol{P}}\right)\left(\sigma_{2} \cdot \hat{\boldsymbol{P}}\right)-\left(\sigma_{1} \cdot \sigma_{2}\right), \\
S_{12}^{\prime \prime}=\frac{3}{2}\left(\sigma_{1} \cdot \hat{\boldsymbol{R}}\right)\left(\sigma_{2} \cdot \hat{\boldsymbol{P}}\right)+\frac{3}{2}\left(\sigma_{2} \cdot \hat{\boldsymbol{R}}\right)\left(\sigma_{1} \cdot \hat{\boldsymbol{P}}\right)-(\hat{\boldsymbol{R}} \cdot \hat{\boldsymbol{P}})\left(\sigma_{1} \cdot \sigma_{2}\right),
\end{gathered}
$$

in terms of unit vectors $\hat{\boldsymbol{R}}, \hat{\boldsymbol{P}}$ (e.g. $\hat{\boldsymbol{R}}=\boldsymbol{R} / \boldsymbol{R}$ ). For the gaussian kernels where the replacement

$$
b / r \rightarrow \exp \left(-\chi^{2} r^{2}\right)
$$

is made in the orbital function $F(r)$ of the interaction (see eq. (5)), the Wigner transforms will be renamed $\hat{G}_{\mathrm{W}}^{\Omega}\left(\boldsymbol{R}, \boldsymbol{P} ; \chi^{2}\right)$, and the $\mathscr{G}_{\mathrm{w}}^{\Omega}\left(\boldsymbol{R}, \boldsymbol{P} ; \chi^{2}\right)$ have the form

$$
\mathscr{G}_{\mathrm{w}}^{\Omega}\left(\boldsymbol{R}, \boldsymbol{P} ; \chi^{2}\right)=-\frac{\pi}{2} \frac{\hbar}{m c} \frac{1}{b^{2}} \frac{1}{\sqrt{3}}\left(\frac{4 \sqrt{3}}{13}\right)^{3 / 2}\left(\frac{13}{6\left(1+2 \chi^{2} b^{2}\right)+\chi^{2} b^{2}}\right)^{3 / 2} g_{\mathrm{w}}^{\Omega}\left(\boldsymbol{R}, \boldsymbol{P} ; \chi^{2}\right)
$$

with

$$
\begin{aligned}
& g_{\mathrm{w}}^{\Omega}\left(\boldsymbol{R}, \boldsymbol{P} ; \chi^{2}\right)=\left(4 / D_{\mathrm{W}}\right)^{3 / 2} \exp \left[-\eta(R / b)^{2}-\zeta(b P / \hbar)^{2}\right] \cos [\xi(\boldsymbol{R} \cdot \boldsymbol{P}) / \hbar] \\
& \quad \times\left\{\begin{array} { l } 
{ v ^ { ( 0 ) } + v ^ { ( 2 ) } ( R / b ) ^ { 2 } + u ^ { ( c ) } ( b P / \hbar ) ^ { 2 } + u ^ { ( s ) } ( \boldsymbol { R } \cdot \boldsymbol { P } / \hbar ) \operatorname { t a n } [ \xi ( \boldsymbol { R } \cdot \boldsymbol { P } / \hbar ) ] } \\
{ v ^ { L S } } \\
{ v ^ { ( 2 ) } ( R / b ) ^ { 2 } ; u ^ { ( c ) } ( b P / \hbar ) ^ { 2 } ; u ^ { ( s ) } | \boldsymbol { R } | | \boldsymbol { P } | / \hbar }
\end{array} \text { for } \left\{\begin{array}{l}
\mathrm{C} \\
L S \\
\mathrm{~T} ; \mathrm{T}^{\prime} ; \mathrm{T}^{\prime \prime}
\end{array}\right.\right.
\end{aligned}
$$

For these gaussian kernels of simple $q \bar{q}$ mesonic exchange type, the coefficients $\eta$, $\zeta, \xi, D_{\mathrm{w}}$, and the potential constants $v^{(0)}, v^{(2)}, u^{(\mathrm{c})}, \boldsymbol{u}^{(\mathrm{s})}, v^{L S}$ are given in table 1 as functions of the parameter $\kappa=\chi^{2} b^{2} /\left(1+2 \chi^{2} b^{2}\right)$. Note that the long-range terms of the interaction (given by $\chi^{2} \rightarrow 0$ contributions) are given by the M-type interaction 
terms only; and in this limit the coefficients $v^{(0)}, v^{(2)}$ of the $P$-independent terms are of exchange type $P_{37}, i=3$ only; while in this limit $v^{L S}$ gets contributions from both $i=1,2$ and $i=3$ exchange types.

Finally, the Wigner transforms of the coupling kernels of simple color-singlet $q \bar{q}$ mesonic exchange type can be assembled by combining eqs. (2), (7), (26), (31)-(33), and (11). They have the form

$$
\begin{aligned}
G_{\mathrm{W}}(\boldsymbol{R}, \boldsymbol{P})= & G_{\mathrm{W}}^{\mathrm{C}-\mathrm{SS}}(\boldsymbol{R}, \boldsymbol{P})+G_{\mathrm{W}}^{L S}(\boldsymbol{R}, \boldsymbol{P})(\boldsymbol{L} \cdot \boldsymbol{S}) \\
& +G_{\mathrm{w}}^{\mathrm{T}}(\boldsymbol{R}, \boldsymbol{P}) S_{12}+G_{\mathrm{W}}^{\mathrm{T}}(\boldsymbol{R}, \boldsymbol{P}) S_{12}^{\prime}+G_{\mathrm{w}}^{\mathrm{T}^{\prime \prime}}(\boldsymbol{R}, \boldsymbol{P}) S_{12}^{\prime \prime} .
\end{aligned}
$$

Their more explicit dependence on nucleon spin and isospin operators follows from the $\sigma, \tau$-dependent terms of eq. (28b), to give

$$
\begin{aligned}
G_{\mathrm{W}}^{\mathrm{C}-\mathrm{SS}}(\boldsymbol{R}, \boldsymbol{P})= & \left(\boldsymbol{\sigma}_{1} \cdot \boldsymbol{\sigma}_{2}\right) G_{\mathrm{W}}^{\eta \mathrm{SS}}(\boldsymbol{R}, \boldsymbol{P})+\left(\boldsymbol{\sigma}_{1} \cdot \boldsymbol{\sigma}_{2}\right)\left(\tau_{1} \cdot \tau_{2}\right) G_{\mathrm{W}}^{\pi \mathrm{SS}}(\boldsymbol{R}, \boldsymbol{P}) \\
& +\left[G_{\mathrm{W}}^{\omega \mathrm{C}}(\boldsymbol{R}, \boldsymbol{P})+\left(\boldsymbol{\sigma}_{1} \cdot \boldsymbol{\sigma}_{2}\right) G_{\mathrm{W}}^{\omega \mathrm{SS}}(\boldsymbol{R}, \boldsymbol{P})\right] \\
& +\left(\tau_{1} \cdot \tau_{2}\right)\left[G_{\mathrm{W}}^{\rho \mathrm{C}}(\boldsymbol{R}, \boldsymbol{P})+\left(\boldsymbol{\sigma}_{1} \cdot \boldsymbol{\sigma}_{2}\right) G_{\mathrm{W}}^{\rho \mathrm{SS}}(\boldsymbol{R}, \boldsymbol{P})\right] \\
G_{\mathrm{W}}^{L S}(\boldsymbol{R}, \boldsymbol{P})= & G_{\mathrm{W}}^{\omega L S}(\boldsymbol{R}, \boldsymbol{P})+\left(\boldsymbol{\tau}_{1} \cdot \tau_{2}\right) G_{\mathrm{W}}^{\rho L S}(\boldsymbol{R}, \boldsymbol{P}) \\
G_{\mathrm{W}}^{\mathrm{T}}(\boldsymbol{R}, \boldsymbol{P})= & G_{\mathrm{W}}^{n \mathrm{~T}}(\boldsymbol{R}, \boldsymbol{P})+\left(\boldsymbol{\tau}_{1} \cdot \tau_{2}\right) G_{\mathrm{W}}^{\pi \mathrm{T}}(\boldsymbol{R}, \boldsymbol{P})+G_{\mathrm{W}}^{\omega \mathrm{T}}(\boldsymbol{R}, \boldsymbol{P}) \\
& +\left(\boldsymbol{\tau}_{1} \cdot \tau_{2}\right) G_{\mathrm{W}}^{\rho \mathrm{T}}(\boldsymbol{R}, \boldsymbol{P})
\end{aligned}
$$

with similar relations for $G_{\mathrm{W}}^{\mathrm{T}^{\prime}}$ and $G_{\mathrm{W}}^{\mathrm{T}}$. Eqs. (35) show that these have precisely the $\sigma, \tau$ dependence of the conventional meson exchange potentials. For the vector mesons, the central term contains both spin-independent terms, denoted by $\mathrm{C}$, and spin-spin terms denoted by SS, following standard OBEP language. For the pseudoscalar mesons the central term is of pure SS variety. The functions $G_{\mathrm{W}}^{\beta \Omega}(\boldsymbol{R}, \boldsymbol{P})$ are given by

$$
\begin{aligned}
G_{\mathrm{W}}^{\beta \Omega}(\boldsymbol{R}, \boldsymbol{P})= & \alpha_{\mathrm{s}} x^{2} m c^{2} \frac{4}{\sqrt{3 \pi}}\left(\frac{4 \sqrt{3}}{13}\right)^{3 / 2} \sum_{\sigma=\mathrm{D}, \mathrm{M}} \sum_{i=1}^{3} \hat{X}_{i}^{O \beta \Omega} \\
& \times \int_{0}^{\infty} \mathrm{d}\left(b_{\chi}\right)\left(\frac{13}{6\left(1+2 \chi^{2} b^{2}\right)+\chi^{2} b^{2}}\right)^{3 / 2} \mathrm{~g}_{(37), \mathrm{i}_{\mathrm{w}}}^{O \Omega}\left(\boldsymbol{R}, \boldsymbol{P} ; \chi^{2}\right),
\end{aligned}
$$

where the $g_{\mathrm{w}}^{\Omega}$ are given by eq. (33) and table 1 , and the $\hat{X}$ contain the $\sigma, \tau$ independent factors of the $X_{P_{37}, i}^{\sigma, \alpha \Omega}$ of eq. (28) and additional $\alpha$-dependent factors. With the shorthand notation

$$
\begin{gathered}
\alpha \equiv\left[\frac{1}{2} \frac{1}{2}(00) S_{3} T_{3}(00)\right] S_{23 \frac{1}{2}} \equiv \beta S_{23}, \\
\hat{c}_{\beta S_{23}} \equiv \frac{1}{\left[1+\delta_{S_{232} \frac{3}{2}}\right]^{1 / 2}} c_{0}^{3} c_{\alpha} N_{\alpha \alpha}^{-1 / 2} \times\left\{\begin{array}{l}
1 \\
\frac{1}{3}
\end{array}\right\} \quad \text { for }\left\{\begin{array}{l}
T_{3}=0 ; \beta=\eta, \omega \\
T_{3}=1 ; \beta=\pi, \rho,
\end{array}\right.
\end{gathered}
$$


TABLE 1

Coefficients for Wigner transforms of gaussian kernels

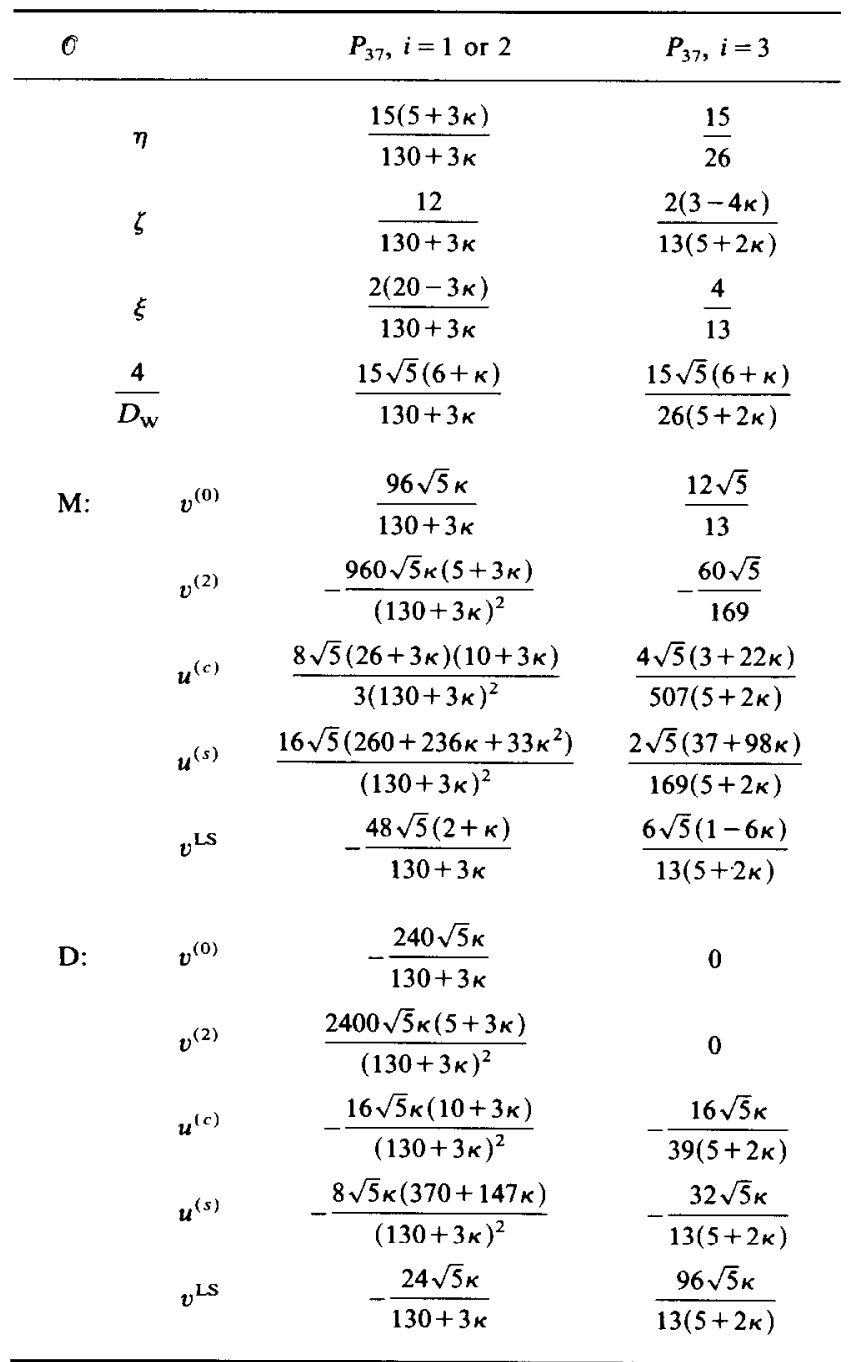

$\kappa=\chi^{2} b^{2} /\left(1+2 b^{2} \chi^{2}\right)$.

the $\hat{X}$ are defined through relations (a) and (b) below:

(a) with $\beta=\eta$ or $\pi$ :

$$
\hat{X}_{i}^{O \beta \mathrm{SS}}=\hat{X}_{i}^{O \beta \mathrm{T}}=\hat{X}^{O \beta \mathrm{T}^{\prime}}=\hat{X}^{O \beta \mathrm{T}^{\prime \prime}}=\hat{c}_{\beta} X_{(34), i}^{O, \beta},
$$

(b) with $\beta=\omega$ or $\rho$ :

$$
\hat{X}_{i}^{O \beta C}=\left(\hat{c}_{\beta \frac{1}{2}}+2 \hat{c}_{\beta \frac{3}{2}}\right)\left(X_{(34), i}^{O, \beta \frac{1}{2}}+2 X_{(34), i}^{O, \beta \frac{3}{3}}\right),
$$




$$
\begin{aligned}
& \hat{X}_{i}^{O \beta S S}=\frac{2}{3}\left(\hat{c}_{\beta \frac{1}{2}}-\hat{c}_{\beta \frac{3}{2}}\right)\left(X_{(34)^{2}, i}^{O, \beta \frac{1}{2}}-X_{(34), i}^{\mathcal{O}, \frac{3}{2}}\right), \\
& \hat{X}_{i}^{O \beta L S}=-\frac{1}{3}\left\{2 \hat{c}_{\beta_{2}^{1}} X_{(34), i}^{O \beta_{1}^{1}}+\hat{c}_{\beta_{2}^{1}} X_{(34), 4}^{O, \beta_{2}^{3}}+\hat{c}_{\beta_{2}^{3}} X_{(34), i}^{O \beta \frac{1}{2}}-4 \hat{c}_{\beta_{2}^{3}} X_{(34), i}^{O, \beta_{2}^{3}}\right\}, \\
& \hat{X}_{i}^{O \beta \mathrm{T}}=\hat{X}_{i}^{O \beta \mathrm{T}^{\prime}}=\hat{X}_{i}^{O \beta \mathrm{T}^{\prime \prime}}=-\frac{1}{2} \hat{X}_{i}^{O \beta S S} \text {. }
\end{aligned}
$$

In eqs. (39) the $X_{(34), i}^{\mathcal{E}, \alpha}$ are the single-nucleon spin, isospin, color factors for the color-singlet $\alpha \equiv \beta S_{23}$ which are tabulated in table 1a of ref. ${ }^{1}$ ).

The $G_{\mathrm{W}}^{\beta \Omega}(\boldsymbol{R}, \boldsymbol{P})$ for these simple color-singlet $(q \overline{\mathrm{q}})$ exchange kernels can be expected to give a good measure for the long-range parts of our effective $N N$ potentials. For low-energy NN scattering the Wigner transforms at $\boldsymbol{P}=0$ are of greatest interest. In this case $T^{\prime}$ and $T^{\prime \prime}$ tensor terms are zero and the remaining terms have the specific form

$$
\begin{aligned}
& G_{\mathrm{W}}^{\beta \Omega}(\boldsymbol{R}, 0)=\alpha_{\mathrm{s}} x^{2} m c^{2} \frac{4}{\sqrt{3 \pi}}\left(\frac{6 \sqrt{15}}{13}\right)^{3 / 2} \exp \left[-\frac{15}{26}\left(\frac{R}{b}\right)^{2}\right] \\
& \times\left\{\begin{array} { l } 
{ f _ { 0 } ^ { \beta \Omega } + ( R / b ) ^ { 2 } f _ { 2 } ^ { \beta \Omega } } \\
{ f _ { 0 } ^ { \beta \Omega } } \\
{ ( R / b ) ^ { 2 } f _ { 2 } ^ { \beta \Omega } }
\end{array} \quad \text { for } \left\{\begin{array}{l}
\Omega=\mathrm{C}, \mathrm{SS} \\
\Omega=L S \\
\Omega=\mathrm{T}
\end{array}\right.\right.
\end{aligned}
$$

with

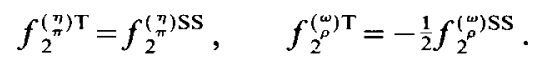

The functions $f_{0}^{\beta \Omega}, f_{2}^{\beta \Omega}$ are given explicitly through relations (a)-(d) below:

(a) for pseudoscalar meson spin-spin terms:

$$
\begin{aligned}
& f_{0^{\pi}}^{\left(\frac{\eta}{\pi}\right) S S}=\hat{c}_{\left(\frac{\eta}{\pi}\right) \frac{1}{3}\left(A_{1}+B\right)}\left(\begin{array}{c}
1 \\
5 \sqrt{\frac{1}{3}}
\end{array}\right), \\
& f_{\left.2^{\frac{\pi}{\pi}}\right) S S}^{\left(\frac{\eta}{c_{(j)}}\right)}\left(-\frac{1}{3}\right)\left(\frac{1}{2} A_{2}+\frac{5}{13} B\right)\left(\begin{array}{c}
1 \\
5 \sqrt{\frac{1}{3}}
\end{array}\right) ;
\end{aligned}
$$

(b) for vector-meson central terms:

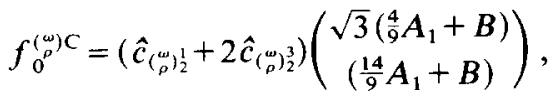

$$
\begin{aligned}
& f_{2}^{(\omega) C}=\left(\hat{c}_{\left(\begin{array}{c}
\omega \\
\rho
\end{array}\right) \frac{1}{2}}^{(\omega)}+2 \hat{c}_{\left.\left(\begin{array}{l}
\omega \\
\rho
\end{array}\right) \frac{3}{2}\right)}\left(\begin{array}{c}
-\sqrt{3}\left(\frac{2}{9} A_{2}+\frac{5}{13} B\right) \\
-\left(\frac{7}{9} A_{2}+\frac{5}{13} B\right)
\end{array}\right) ;\right.
\end{aligned}
$$

(c) for vector-meson spin-spin terms:

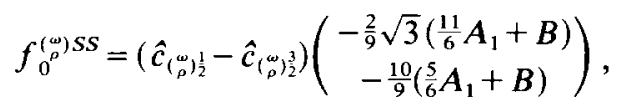


(d) for vector-meson LS terms:

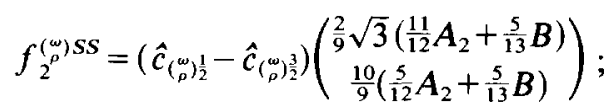

$$
\begin{aligned}
& f_{0}^{\omega L S}=-\hat{c}_{\omega_{2} \frac{1}{2}} \frac{1}{15 \sqrt{3}}\left(\frac{2}{13} A_{0}+\frac{11 \times 17}{4 \times 13} A_{1}+\frac{17}{3} B\right) \\
& +\hat{c}_{\omega^{\frac{3}{2}}} \frac{1}{3 \sqrt{3}}\left(\frac{1}{13} A_{0}+\frac{63}{26} A_{1}+\frac{11}{6} B\right), \\
& f_{0}^{p L S}=\hat{c}_{\rho^{\frac{1}{4}}} \frac{1}{45}\left(\frac{2}{13} A_{0}+\frac{11 \times 17}{4 \times 13} A_{1}-B\right) \\
& +\hat{c}_{\rho_{2} \frac{3}{45}}\left(A_{0}+\frac{63}{2} A_{1}+\frac{37}{2} B\right) \text {; }
\end{aligned}
$$

where the $\hat{c}_{\beta S_{23}}$ are defined in eq. (38), and

$$
\begin{aligned}
A_{0} & =\left(\frac{130}{263}\right)^{1 / 2} 16 \sqrt{5} h_{0}(a), \\
A_{1} & =\left(\frac{130}{263}\right)^{1 / 2} \frac{16}{263} \sqrt{5} h_{1}(a), \\
A_{2} & =\left(\frac{130}{263}\right)^{1 / 2} \frac{160}{13 \times 263} \sqrt{5}\left[h_{1}(a)+\frac{45}{263} h_{2}(a)\right], \\
B & =\frac{10}{13} \sqrt{\frac{1}{3}},
\end{aligned}
$$

with

$$
\begin{gathered}
a=\frac{130}{263}\left(\frac{15}{26}\right)^{2}(R / b)^{2}, \\
h_{0}(a)=\frac{1}{2} \sqrt{\frac{\pi}{a}} \operatorname{erf}(\sqrt{a}), \\
h_{1}(a)=\frac{3}{2 a}\left[\frac{1}{2} \sqrt{\frac{\pi}{a}} \operatorname{erf}(\sqrt{a})-\mathrm{e}^{-a}\right], \\
h_{2}(a)=\frac{5}{2 a}\left[\left(\frac{3}{2 a}\right) \frac{1}{2} \sqrt{\frac{\pi}{a}} \operatorname{erf}(\sqrt{a})-\left(1+\frac{3}{2 a}\right) \mathrm{e}^{-a}\right],
\end{gathered}
$$

where

$$
\operatorname{erf}(x)=\frac{2}{\sqrt{\pi}} \int_{0}^{x} \mathrm{~d} t \mathrm{e}^{-t^{2}},
$$

and the $h_{n}$ are defined such that $h_{n}(0)=1$. Note that the $A_{n}$ terms contain a weak $R$-dependent falloff in the long-range limit whereas $B$ is independent of $R$ and arises from the $\chi^{2} \rightarrow 0$ long-range terms discussed in connection with table 1 .

\section{Comparison with conventional OBEP's}

The Wigner transforms of the simple color-singlet $q \bar{q}$ exchange terms, specifically the $G_{\mathrm{w}}^{\beta \Omega}(\boldsymbol{R}, 0)$ of eqs. (35) and (40), are identified as the effective meson exchange 
potentials of our quark model of the two-nucleon system. Eqs. (35) show that these are in 1:1 correspondence with conventional OBEP's as far as their dependence on nucleon $\left(\sigma_{1} \cdot \sigma_{2}\right)$ and $\left(\tau_{1} \cdot \tau_{2}\right)$ factors are concerned. They also have the well-known characteristic features of OBEP's with respect to the relative importance and signs of tensor- and spin-spin-type central terms. Specifically, the tensor force has the opposite sign for pseudoscalar and vector-meson exchange potentials whereas the spin-spin-type central terms all have the same sign. The simple qã exchange potentials corresponding to pseudoscalar meson exchange have only SS-type central terms and no $L S$ terms. In our quark model, the presence of both C and SS terms, as well as $L S$ terms, for the q $\bar{q}$ exchange potentials corresponding to vector-meson exchange is related to the fact that these gain contributions from two types of spin-dependent terms, with $S_{23}=\frac{1}{2}$ and $\frac{3}{2}$ (note the difference between eqs. (39b) and (39a)). Figs. 7-11 show that the $G_{\mathrm{W}}^{\beta \Omega}$ are in general also in good quantitative agreement with the conventional one-meson exchange potentials $V^{\beta \Omega}$ in the $R \geqslant$ $1.2 \mathrm{fm}$ range and have the general radial characteristics of the $V^{\beta \Omega}$ over an even wider range. For the conventional meson exchange potentials we have chosen the on-shell, nonrelativistic meson potentials of Holinde and Mundelius ${ }^{4}$ ), in $P^{2}=0$ approximation, to be directly comparable to the $G_{\mathrm{W}}^{\beta \Omega}$ evaluated at $\boldsymbol{P}=0$. Specifically, we have chosen the meson potentials, given in momentum representation, by eq. (2.25) of ref. ${ }^{4}$ ) for pseudoscalar mesons and by eq. (A.5) of ref. $\left.{ }^{4}\right)$ for vector mesons. These are regularized by the momentum-dependent form factors of the coupling constants

$$
g\left(k^{2}\right)=g(0)\left(\frac{\Lambda^{2}}{\Lambda^{2}+k^{2}}\right)^{n}
$$

with dipole approximation, $n=1$, for pseudoscalar mesons, and $n=\frac{3}{2}$ for vector mesons, as suggested by Holinde ${ }^{5}$ ). A common cutoff parameter $\Lambda=1530 \mathrm{MeV}$ has been used for all mesons ${ }^{5}$ ). The meson masses and coupling constants, (including the tensor to vector coupling ratio $f_{\mathrm{NN} \rho} / g_{\mathrm{NN} \rho}$ ), used to evaluate the $V^{\beta \Omega}(R)$ are those of table 1 of ref. $\left.^{5}\right)$. Since the quark-model parameters of ref. ${ }^{1}$ ) fit the experimental vector-meson coupling constants, in particular the combination $\left(g_{\mathrm{NN} \rho}+f_{\mathrm{NN} \rho}\right)$, the $G_{\mathrm{W}}^{\beta \Omega}$ and $V^{\beta \Omega}$ are essentially based on the same set of experimental numbers. The extreme short-range parts of the $V^{\beta \Omega}$ are not shown in figs. 7-11, although in some cases, specifically the $V^{\beta T}$, there are additional changes in sign at extreme short range which are not to be taken seriously with the above high $-k^{2}$ form-factor parameterization.

Figs. 7-11 show a remarkable agreement between the long-range characteristics of $G_{\mathrm{W}}^{\beta \Omega}$ and $V^{\beta \Omega 2}$. The extreme long-range falloff of our central pion potential $G_{\mathrm{W}}^{\pi \mathrm{sS}}$ does not match the Yukawa tail of $V^{\pi \mathrm{SS}}$; but this is to be expected from a quark model with gaussian 0s internal wave functions. In addition, our quark-model pion tensor term is too weak. At $R=1.2 \mathrm{fm}, G_{\mathrm{W}}^{\pi \mathrm{T}}$ is approximately $\frac{1}{3}$ of $V^{\pi \mathrm{T}}$. This is in agreement with the fact that the $\pi \mathrm{NN}$ coupling constant, $g_{\pi \mathrm{NN}}^{2}$, predicted by our 


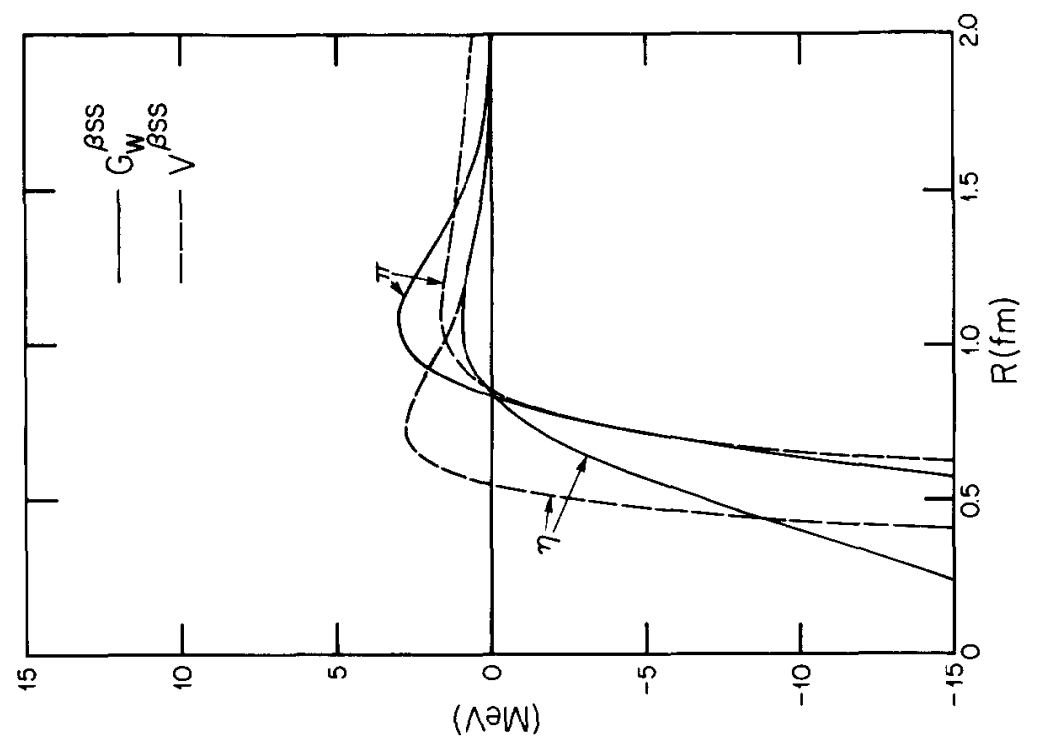

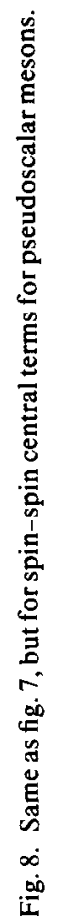
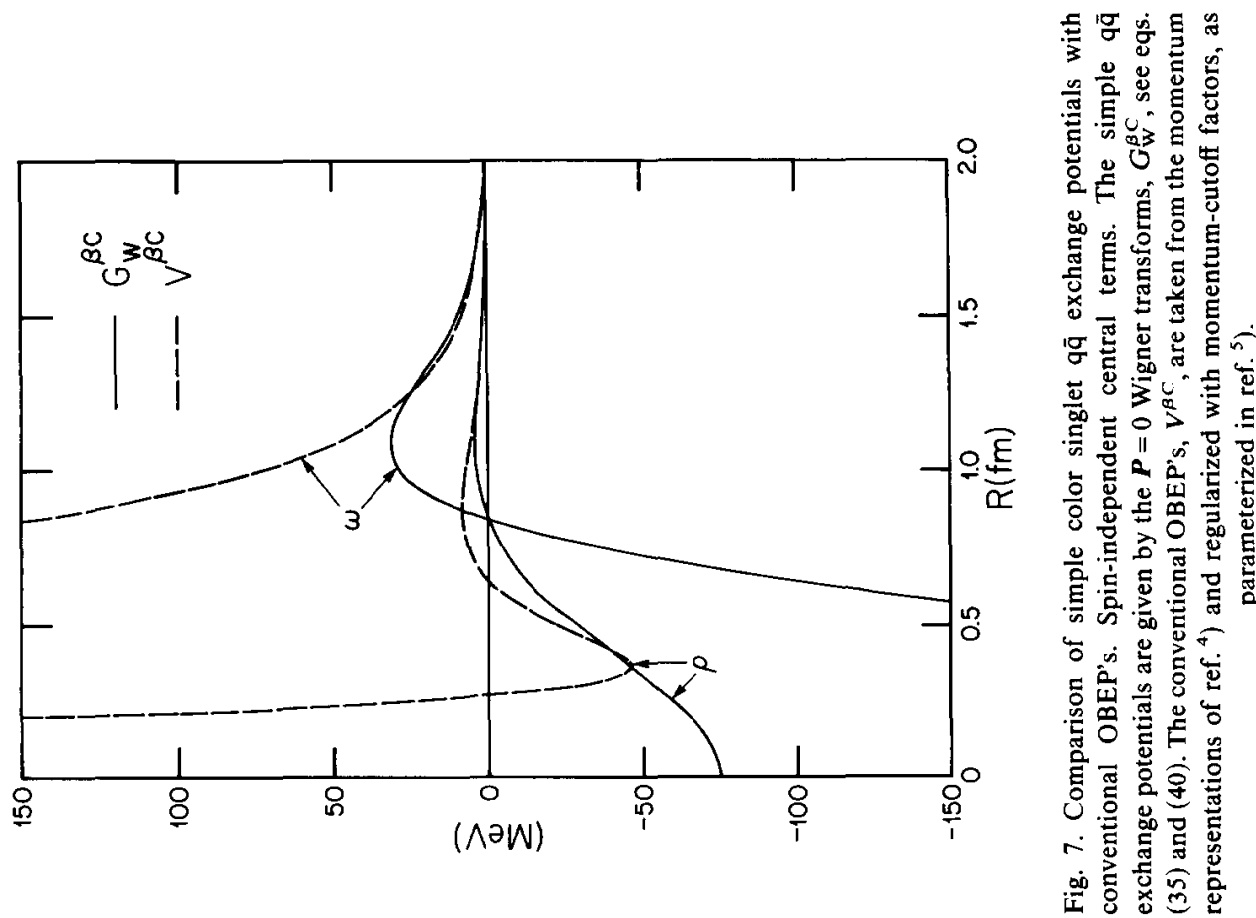

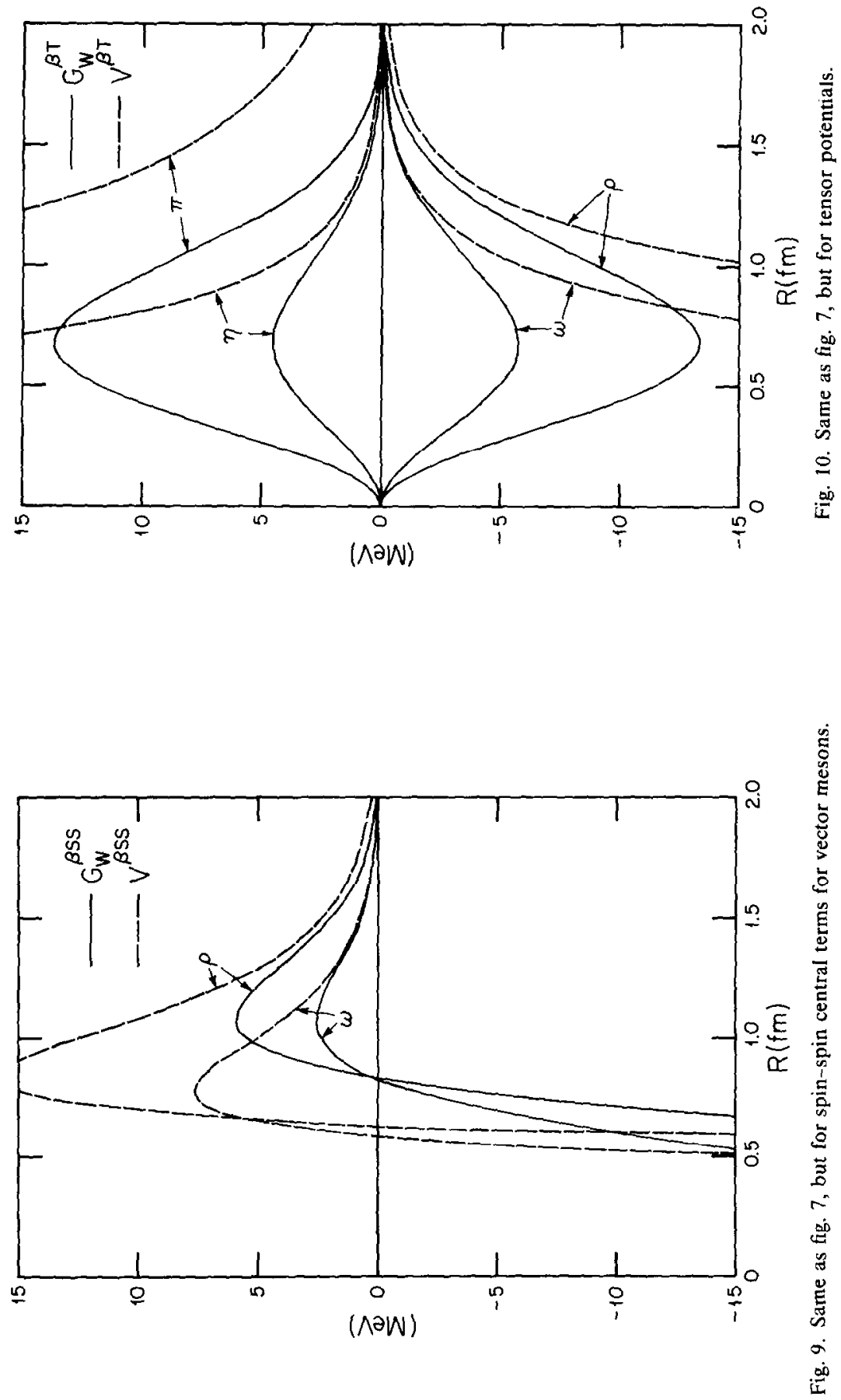


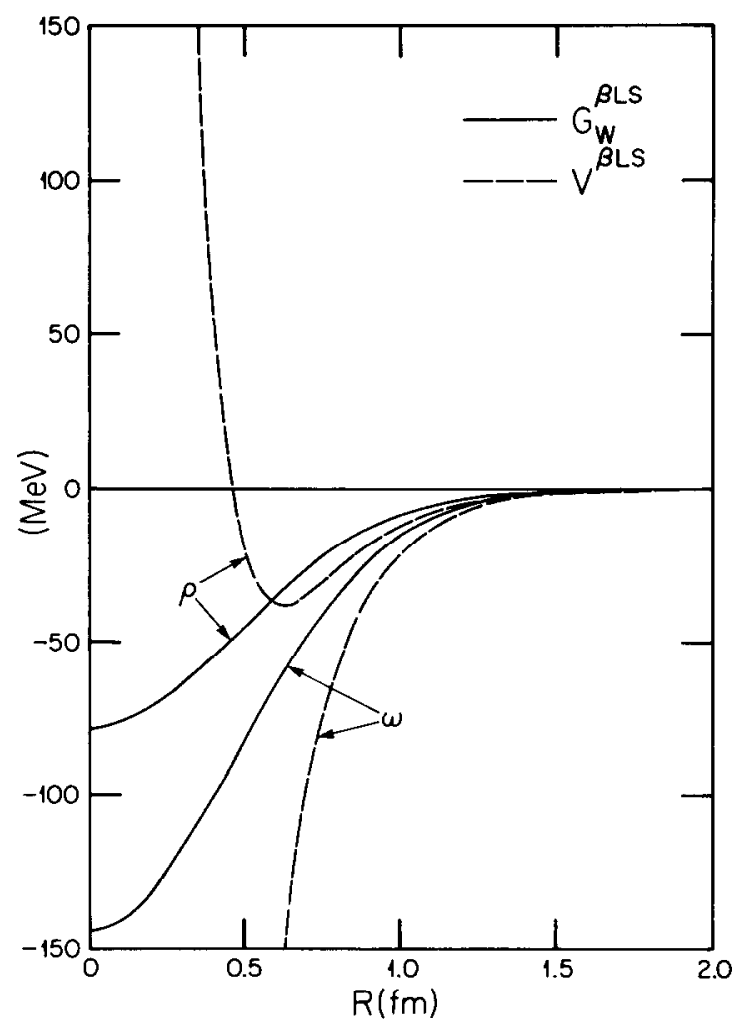

Fig. 11. Same as fig. 7, but for the spin-orbit potentials.

model, is too weak by a factor of $\sim \frac{1}{3}\left[\right.$ see ref. $\left.\left.^{1}\right)\right]$. With the exception of the long-range parts of the pion potentials, the $G_{\mathrm{W}}^{\beta \Omega}$ and $V^{\beta \Omega}$ are in remarkably good agreement for $R \geqslant 1.2 \mathrm{fm}$. In addition, they have the same qualitative characteristics over a much wider range. This can be seen for the central SS terms from figs. 8 and 9 , although we note that the zeros of the $G_{\mathrm{W}}^{\beta S S}$ are pushed to larger $R$-values by $0.2-0.3 \mathrm{fm}$ relative to those of $V^{\beta S S}$ for $\beta=\eta, \omega$ and $\rho$. Fig. 7 shows that the most significant qualitative difference between the $\mathrm{q} \overline{\mathrm{q}}$ exchange potentials $G_{\mathrm{W}}^{\beta \Omega}$ and the conventional meson exchange potentials $V^{\beta \Omega}$ occurs in the spin-independent central part of the $\omega$-exchange potential. The steep repulsive rise in the central part of the conventional $\omega$-exchange potential in the $R \leqslant 1 \mathrm{fm}$ range is missing in the corresponding $\mathrm{q} \overline{\mathrm{q}}$ exchange potential. The latter changes sign, with a zero at $\sim 0.83 \mathrm{fm}$, and becomes attractive at extremely short range with a minimum of $-583 \mathrm{MeV}$ at $R=0$. [This compares with a much steeper attractive minimum of $\sim 1.2 \mathrm{GeV}$ for the full " $\mathrm{N} \omega$ " potential if all 25 exchange terms are included, cf. fig. 10 of ref. ${ }^{1}$ ).] It should be noted that a change of sign at $\sim 0.83 \mathrm{fm}$ is common to all central potentials of simple color-singlet $q \bar{q}$ exchange type. This is related to the nature of the essentially common second-degree polynomial multiplier shared by all central $G_{\mathrm{w}}^{\beta \Omega}$, so that 
this is not a unique feature of the $\omega$-exchange term. In this connection it is also interesting to note that Holinde ${ }^{9}$ ) seeks the origin of the short-range repulsion of the "phenomenological $\omega$-potential" in the short-range repulsive term arising through the quark-gluon exchange mechanism of the (3q)-(3q) model, since only the outerrange parts of the meson exchange potentials are considered as theoretically "well founded". Finally, it is interesting to note that phenomenological models, in which a short-range repulsive potential, derived from quark exchange kernels in a (3q)-(3q) model, is augmented by longer-range mesonic potentials, introduced into the model "by hand", can fit the experimental NN scattering data only if the $\omega$-repulsion is "turned off". [See ref. ${ }^{10}$ ) as an example of such calculations.]

Fig. 11 shows that the $\rho$ and $\omega L S$ potentials of the simple q $\bar{q}$ exchange type are in reasonable quantitative agreement with the conventional meson exchange potentials for $R \geqslant 0.8 \mathrm{fm}$. It is to be noted, in particular, that the $L S$ attraction is of the same order of magnitude or even larger than the weak attractive $L S$ potential generated by the (3q)-(3q) components of the two-nucleon system [see refs. $\left.{ }^{11-13}\right)$ ]. Before definite conclusions can be drawn for the $R<0.8 \mathrm{fm}$ region the full $L S$ potentials must be calculated (including all 25 types of exchange terms). However, the results of fig. 11 indicate that the cross-terms in the quark exchange kernels, which arise from the coupling of the (3q)-(3q) components of the two-nucleon wave function to the $(3 q)-(3 q)(q \bar{q})$ components, are of major importance for the $L S$ potentials. Conclusions drawn from $L S$ potentials derived from simple (3q)-(3q) models may therefore be open to question. It is interesting to note that $L S$ potentials from simple (3q)-(3q) models have been combined with additional phenomenological $L S$ potentials of meson-exchange type in a recent application ${ }^{14}$ ).

Since the short-range parts of conventional meson exchange potentials are very sensitive to the exact form of the high-momentum cutoff function in the assumed form factor of eq. (41), it is also of interest to note that our model predicts such form factors; see eq. (69) or ref. ${ }^{1}$ ). Although there are slight differences in the $\boldsymbol{k}^{2}$ falloff for different mesons these are approximated very well (with better than $95 \%$ accuracy over the $k=0-6 \mathrm{fm}^{-1}$ range) by a simple gaussian falloff, $\exp \left(-0.047 \boldsymbol{k}^{2}\right)$, for all mesons. The nature of this falloff is of course related to the gaussian character of the internal wave functions of our model and again is not to be taken seriously for very high $\boldsymbol{k}^{2}$. Meson exchange potentials in which the form factor of eq. (41) is replaced by this simple gaussian have characteristics very similar to those of both the $G_{\mathrm{W}}^{\beta \Omega}$ and $V^{\beta \Omega}$ of figs. 7-11, including a strong short-range repulsive $\omega \mathrm{C}$ term. However, the agreement between $G_{\mathrm{W}}^{\beta \Omega}$ and $V^{\beta \Omega}$ in the $R \geqslant 1.2 \mathrm{fm}$ range is best if the $V^{\beta \Omega}$ are evaluated with the experimentally-based form factors of ref. ${ }^{5}$ ) and eq. (41).

Despite the good agreement between the $G_{\mathrm{W}}^{\beta \Omega}$ and $V^{\beta \Omega}$ for $R \geqslant 1.2 \mathrm{fm}$ and their similarities in the $R \sim 1 \mathrm{fm}$ region, it should be emphasized that there is much cancellation in this region between the various mesonic contributors to the effective NN potential in a particular NN channel. The simple color-singlet $q \bar{q}$ exchange 
potentials can thus not be expected to be a good approximation in the $R \sim 1 \mathrm{fm}$ region where more complicated exchange processes already dominate the full NN potentials. Exchange terms of type $P_{36} P_{37}$ with $i=1-3$, in particular, have been found to be important in this region.

Finally, it may be interesting to compare the results of the present investigation and the philosophy underlying our model with that of other models in which meson fields are coupled to the quark degrees of freedom ${ }^{2}$ ) through a meson quark-current interaction with coupling constant determined by the PCAC relation. The work of Shimizu ${ }^{15}$ ) is of particular relevance for the present study. Shimizu studies the effect of quark antisymmetrization on the one-pion exchange potential derived from such a model. His long-range potential, of conventional $\left(\sigma_{1} \cdot \sigma_{2}\right)\left(\tau_{1} \cdot \tau_{2}\right)$ type, is qualitatively similar to the $G_{\mathrm{W}}^{\pi \mathrm{SS}}$ and $V^{\pi \text { sS }}$ of fig. 8. However, he also predicts that quark antisymmetrization leads to a strong short-range spin, isospin-independent central repulsive component in the one pion exchange potential. Neither the simple $q \bar{q}$ exchange potential of pionic type nor the full $\mathrm{N} \pi$ potential of our model contains such terms. Fig. 9 of ref. ${ }^{1}$ ) shows that the full $\mathrm{N} \pi$ potential, including all 25 of the possible exchange terms in the coupling kernel of $N \pi(3 q)(q \bar{q})$ type, shows a relatively strong attractive component as $R \rightarrow 0$. Since it is the aim of the present quark model, with q $\bar{q}$ excitations explicitly incorporated into the model space, to treat the quark degrees of frecdom in baryons and mesons on an equal footing, quark antisymmetrization effects are fully included. Such models should ultimately bridge the gap between models in which mesons are treated as separate entities and models in which both baryons and mesons are treated from a more fundamental viewpoint.

\section{Summary}

In a recent quark-model study of the $\mathrm{NN}$ interaction ${ }^{1}$ ), we explicitly incorporated the quark-antiquark excitations inherent in the quark-gluon interaction lagrangian into the model space to study the effects of such excitations on the NN interactions. The admixture of a specific $(3 q)(q \bar{q})$ component into the wave function of a single nucleon leads to important contributions to the quark exchange kernels of the two-nucleon system. When converted into effective potentials through a localmomentum approximation using the Wigner transforms of these kernels, these coupling terms lead to important short-range potentials whose net effect is to cancel much of the repulsive core height arising from the dominant (3q)-(3q) component of the two-nucleon wave function. The present investigation has focused on the long-range terms of these coupling kernels. It is shown that the long-range parts of the resultant potentials arise almost entirely through two of the 25 possible types of exchange terms. These two exchange terms correspond to a simple exchange of a $q \bar{q}$ pair between the two nucleons and contribute only through coupling terms for which the $(3 q)$ component has the quantum numbers of a nucleon and the $(q \bar{q})$ 


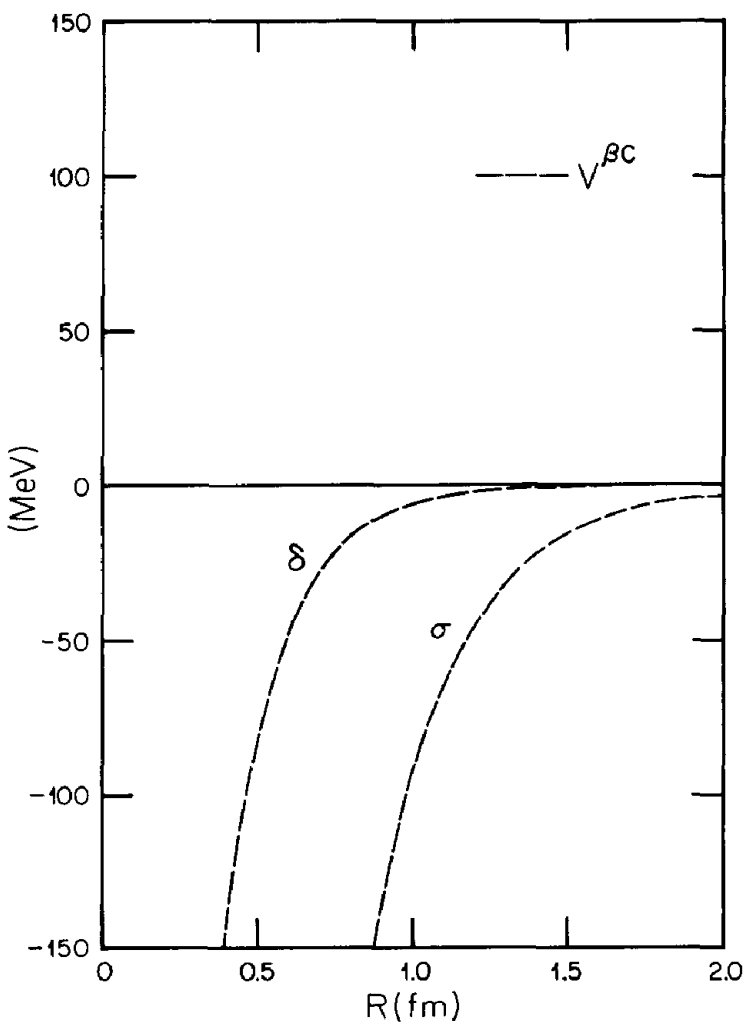

Fig. 12. OBEP's, scalar mesons ( $\sigma$-meson, $T=0 ; \delta$-meson, $T=1$ ).
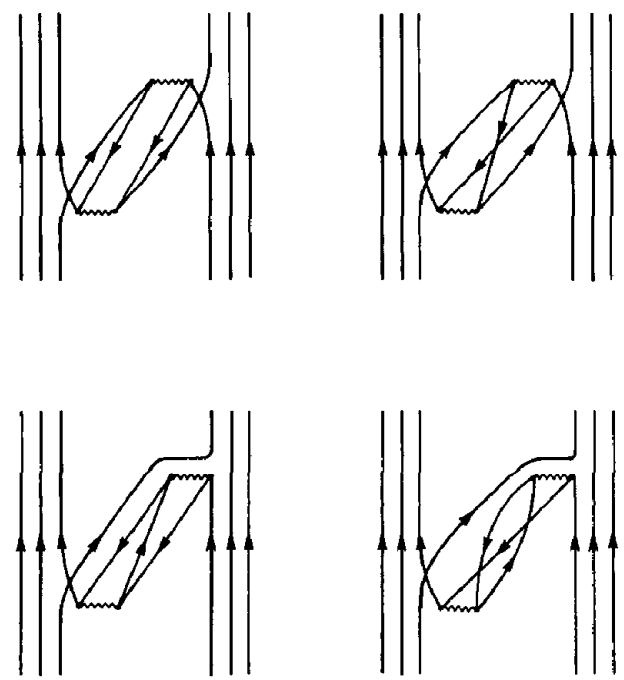

Fig. 13. Quark exchange diagrams generated by RPA-type off-shell terms of the Fermi-Breit quark-gluon interaction. 
component the color-singlet character of a real pseudoscalar or vector meson. The potentials arising from these simple color-singlet $q \bar{q}$ exchanges have the characteristics of conventional one-meson exchange potentials as far as their dependence on nucleon $\left(\sigma_{1} \cdot \sigma_{2}\right)$ and $\left(\tau_{1} \cdot \tau_{2}\right)$ factors and their $L S$ and tensor properties are concerned. They are also in remarkably good agreement with conventional OBEP's in the $R \geqslant 1.2 \mathrm{fm}$ range and have the same qualitative radial characteristics over an even wider range. The one exception is the spin-independent central term of the $\omega$-exchange potential. Although $V^{\omega C}$ and $G_{\mathrm{w}}^{\omega C}$ are in remarkably good agreement for $R \geqslant 1.2 \mathrm{fm}$ the $\mathrm{q} \overline{\mathrm{q}}$ exchange potential does not have the steep short-range repulsive rise of the conventional $\omega$-exchange potential but becomes attractive at $R<0.8 \mathrm{fm}$. Since the quark model instead gains a repulsive core through the $(3 q)-(3 q)$ components of the two-nucleon wave function, this must be considered a desirable feature of our model.

In ref. ${ }^{1}$ ) it was also shown that the effective NN potentials gain a weak mediumrange attractive part through the $\mathrm{q} \overline{\mathrm{q}}$ excitations. However, this attractive part is too weak to fit the low-energy S-wave phase shifts. In the conventional one-meson exchange potentials an important medium-range attraction is introduced through a $\sigma$-potential. Fig. 12 shows the conventional meson-theory $\sigma$ - and $\delta$-potentials using the parameters of refs. ${ }^{4,5}$ ) and the momentum-cutoff form factor of eq. (41), again with $A=1530 \mathrm{MeV}$ and $n=1$. The figure is included to show that the $\sigma$-potential is vital for medium-range attraction in the conventional OBEP picture. Since the $(\mathrm{q} \overline{\mathbf{q}})$ excitations are incorporated into the quark model in ref. $\left.{ }^{1}\right)$ through an improved wave function of an isolated nucleon, the $q \bar{q}$ excitations of the model do not include scalar mesons, and potentials of $\sigma$-meson exchange type are so far missing in the quark model. However, besides the pseudoscalar and vector $q \bar{q}$ excitations inherent in the quark-gluon interaction lagrangian, there are additional $(\mathrm{q} \overline{\mathrm{q}})(\mathrm{q} \overline{\mathrm{q}})$ excitations which can carry the quantum numbers of a $\sigma$-meson. Such $\sigma$-mesonic effects can thus be incorporated into our quark model of the $\mathrm{NN}$ interaction through the $(q \bar{q})(q \tilde{q})$ excitations generated by RPA-type off-shell terms in the Fermi-Breit quark-gluon interaction. Fig. 13, which is to be compared with fig. 1a, is included to show that such excitations can account for $\sigma$-exchange effects. Preliminary calculations show that the $\sigma \mathrm{N}$ coupling constants arising from such exchange terms are of the right order of magnitude. Details will be presented in a future study.

\section{References}

1) Y. Fujiwara and K.T. Hecht, Nucl. Phys. A444 (1985) 541 [see this reference for a guide to the earlier literature]

2) G.E. Brown and M. Rho, Phys. Lett. 82B (1979) 177;

A.W. Thomas, S. Théberge and G.A. Miller, Phys. Rev. D24 (1981) 216;

R. Tegen and W. Weise, Z. Phys. A314 (1983) 357;

H.J. Weber, Phys. Rev. C26 (1982) 2333

3) Y. Fujiwara and Y.C. Tang, University of Minnesota report, UM-RGM2, 1984

4) K. Holinde and H. Mundelius, Nucl. Phys. A364 (1981) 365 
5) K. Holinde, Phys. Reports 68 (1981) 121

6) Y.W. Yu and Z.Y. Zhang, Nucl. Phys. A426 (1984) 557

7) H. Horiuchi, Prog. Theor. Phys. 55 (1976) 1448

8) T.H. Seligman and W. Zahn, J. of Phys. G2 (1976) 79

9) K. Holinde, Nucl. Phys. A415 (1984) 477

10) Y. Suzuki, Nucl. Phys. A444 (1985) 637

11) Y. Suzuki and K.T. Hecht, Nucl. Phys. A420 (1984) 525, and A446 (1985) 749

12) O. Morimatsu, S. Ohta, K. Shimizu and K. Yazaki, Nucl. Phys. A420 (1984) 573

13) F. Wang and C.W. Wong, Nucl. Phys. A438 (1985) 620

14) Y. He, F. Wang and W.C. Wong, UCLA preprint (1985), Nucleon core size and nucleon-nucleon spin-orbit forces

15) K. Shimizu, Phys. Lett. 148B (1984) 418 